\title{
Efficient Homology-directed Repair with Gircular ssDNA Donors
}

\author{
Sukanya Iyer ${ }^{1 *}$ Aamir Mir2* $\$$, Joel Vega-Badillo², Benjamin P. Roscoe ${ }^{1 \dagger}$, Raed
}

Ibraheim², Lihua Julie Zhu' ${ }^{1,3}$, Jooyoung Lee², Pengpeng Liu' ${ }^{1}$, Kevin Luk ${ }^{1}$, Esther

Mintzer ${ }^{1}$, Josias Soares de Brito ${ }^{1}$, Philip D. Zamore ${ }^{2,4}$, Erik J. Sontheimer ${ }^{2,5,6}$ and Scot A.

Wolfe ${ }^{1,6}$

\section{*These authors contributed equally}

Author affiliations

${ }^{I}$ Department of Molecular, Cell and Cancer Biology, University of Massachusetts

Medical School, Worcester, Massachusetts, USA.

${ }^{2} R N A$ Therapeutics Institute, University of Massachusetts Medical School, Worcester, Massachusetts, USA

${ }^{3}$ Program in Bioinformatics and Integrative Biology, University of Massachusetts Medical School, Worcester, Massachusetts, USA

${ }^{4}$ Howard Hughes Medical Institute, University of Massachusetts Medical School, Worcester, Massachusetts, USA.

${ }^{5}$ Program in Molecular Medicine, University of Massachusetts Medical School, Worcester, Massachusetts, USA

${ }^{6}$ Li Weibo Institute for Rare Disease Research, University of Massachusetts Medical School, Worcester, Massachusetts, USA.

Correspondence should be addressed to E.J.S. (erik.sontheimer@umassmed.edu) or S.A.W.(scot.wolfe@umassmed.edu).

§Present address: Inscripta, Inc., 7060 Koll Center Parkway, Suite 312, Pleasanton, CA 94566, USA

$\dagger$ Present address: COGEN Therapeutics, Cambridge, MA, USA

\begin{abstract}
While genome editing has been revolutionized by the advent of CRISPR-based nucleases, difficulties in achieving efficient, nuclease-mediated, homology-directed repair (HDR) still limit many applications. Commonly used DNA donors such as plasmids suffer from low HDR efficiencies in many cell types, as well as integration at unintended sites. In contrast, single-stranded DNA (ssDNA) donors can produce efficient HDR with minimal off-target integration. Here, we describe the use of ssDNA phage to efficiently and inexpensively produce long circular ssDNA (cssDNA) donors. These cssDNA donors serve as efficient HDR templates when used with Cas9 or Cas12a, with integration frequencies superior to linear ssDNA (lssDNA) donors. To evaluate the relative efficiencies of imprecise and precise repair for a suite of different Cas9 or Cas 12a nucleases, we have developed a modified Traffic Light Reporter (TLR) system [TLR-
\end{abstract}


44 Multi-Cas Variant 1 (MCV1)] that permits side-by-side comparisons of different nuclease

45 systems. We used this system to assess editing and HDR efficiencies of different nuclease

46 platforms with distinct DNA donor types. We then extended the analysis of DNA donor types to evaluate efficiencies of fluorescent tag knock-ins at endogenous sites in HEK293T and K562 cells. Our results show that cssDNA templates produce efficient and robust insertion of reporter tags. Targeting efficiency is high, allowing production of biallelic integrants using cssDNA donors. cssDNA donors also outcompete lssDNA donors in template-driven repair at the target site. These data demonstrate that circular donors provide an efficient, cost-effective method to achieve knock-ins in mammalian cell lines.

\section{Introduction}

RNA-guided Cas $9^{1-3}$ and Cas 12 a proteins ${ }^{4,5}$ have provided a facile tool for introducing targeted breaks within genomes. These double-strand breaks (DSBs) can be harnessed to engineer the genome through endogenous DNA repair pathways. Typically, DSBs are precisely repaired via the canonical non-homologous end joining (c-NHEJ) pathway, restoring the original DNA sequence. ${ }^{4}$ However, in the context of a programmable nuclease where DSB generation can reoccur, imprecise DNA repair may produce small insertions and deletions (indels) via c-NHEJ as well as alt-NHEJ pathways. ${ }^{6}$ In contrast to the imprecise nature of these indels, the homology-directed repair (HDR) pathway results in precise rewriting of the genome in a template-dependent manner. ${ }^{7-9} \mathrm{HDR}$ is often utilized in the context of programmable nucleases to introduce specific changes to the genome, such as adding fluorescent tags to proteins ${ }^{10}$ or making a precise therapeutic correction to the desired locus. ${ }^{11-13}$ Given the broad utility of this technology for enabling precise insertions into mammalian genomes, several viral and non-viral approaches for the delivery of donor DNA into mammalian cells have been described. ${ }^{14-17}$ The nature of the template employed for HDR is dictated in part by the length of the desired genomic modification. For short insertions (<200 nt), ssDNA oligonucleotides harboring the mutation, as well as flanking homology arms that range from 35-60 nucleotides, are introduced into cells along with Cas9 protein and guide RNA. ${ }^{15}, 18,19$ When modifications longer than 200bp are desired, double-stranded DNA (dsDNA) templates such as plasmids or PGR products are typically used as donor templates. However, these doublestranded templates are often associated with high cellular toxicity and off-target integration events. ${ }^{20}$ As an alternative to using dsDNA templates as donors for HDR, long ssDNA templates have been reported to have low cytotoxicity and high efficiencies of targeted integration at the site of interest. ${ }^{21,22}$ Consequently, there is considerable interest in developing methods to generate long ssDNA templates to serve as donors for making targeted insertions in mammalian cells. Several recent examples include asymmetric PCR, "Strandase" enzyme-mediated removal of one strand of a linear dsDNA template [Takara Bio USA (catalogue number 632644)], use of pairs of nicking endonucleases followed by gel extraction of resulting ssDNA [Biodynamics Laboratory Inc. (catalogue number DS615) and reverse transcription (RT)-based approaches to generate ssDNA. ${ }^{21-24}$ Most of these approaches require expensive and time-consuming purification steps to ensure complete removal of truncated ssDNA products. With RTbased approaches in particular, it is challenging to generate accurate ssDNA donors 
longer than 3-4 kb, especially in large molar quantities, because of the lack of proofreading activity and the limited processivity of reverse transcriptase enzymes.

As an alternative to these in vitro approaches, we explored the use of circular ssDNA (cssDNA) produced from phagemids as templates for HDR-mediated integration of DNA cassettes. Phagemid vectors have been used to generate ssDNA templates for site-directed mutagenesis ${ }^{25}$, DNA nanotechnology and DNA origami ${ }^{26}$, phage display technology for protein engineering ${ }^{27}$ and as templates for transcription in cell-free systems. ${ }^{28}$ However, to our knowledge, their use as donors for achieving targeted integration of DNA in mammalian cells has not been evaluated.

Here, we show that phagemid-derived cssDNA can be used to insert sequences efficiently and precisely in mammalian cells. We further compared HDR efficiencies obtained with phagemid-sourced cssDNA to those of linear ssDNAs (lssDNAs) generated using a RTbased method ${ }^{22}$ and a streptavidin affinity purification approach with asymmetrically biotinylated PCR amplicons. ${ }^{29}$ To this end, we utilized a redesigned traffic light reporter system to evaluate HDR efficiencies for different forms of donor templates (plasmids, lssDNAs and cssDNAs) when used in conjunction with SpyCas9 or three different Cas12a effectors delivered as ribonucleoproteins (RNPs) in HEK293T and K562 cells. We then compared knock-in yields of linear and circular ssDNA donor templates containing fluorescent reporter tags at four different endogenous sites in the human genome. Finally, we demonstrated the ability of circular ssDNA templates to create biallelic integration of a reporter cassette in different cell lines. Overall, our data show broad utility of cssDNA as donors for genome engineering applications.

\section{Results}

\section{Generating linear and circular ssDNA templates for HDR in mammalian cells}

To address the challenges associated with long ssDNA donor production, we investigated a number of different approaches for generating ssDNA donors, as well as the relative efficiencies of HDR when using the resulting ssDNA products. While most efforts to generate ssDNA donors have focused on linear molecules, we explored the properties of circular ssDNAs as donors for HDR. Phagemids are chimeric vectors that contain plasmid and bacteriophage origins of replication. Upon superinfection of the host bacteria with helper phage to supply the phage DNA replication machinery, one strand of the phagemid vector is packaged into bacteriophage particles and extruded into the media from whence circular ssDNA can be purified ${ }^{30}$ (Supplementary Fig. S1A). Although a standard protocol to purify ssDNA from phagemids yielded reasonable quantities of DNA, we observed the presence of contaminating Escherichia coli genomic DNA in the ssDNA preparation, as reported previously. ${ }^{31}$ To remove contaminating $E$. coli genomic DNA in preparation for donor DNA transfection into mammalian cells, we modified a purification protocol described by Viera and Messing ${ }^{30}$, where we incorporated a DNase I digestion step prior to bacteriophage uncoating and subsequently purified the cssDNA using an anion exchange column. 
To provide a benchmark for aspects of donor DNA production and direct comparison of HDR rates in mammalian cells, we also evaluated two methods for generating lssDNA templates. First, lssDNA was generated using a published RT method (T-lssDNA) in which cDNA is generated by a processive reverse transcriptase such as TGIRT-III. ${ }^{32}$ RTbased approaches (Supplementary Fig. S1B) can be effective for generating ssDNA donors up to $3.5 \mathrm{~kb}$ in length. ${ }^{21,22,33,34}$ However, the reverse transcriptase enzymes used for generating linear ssDNA generally lack proofreading activity ${ }^{35}$, which makes the fidelity of the resulting template a concern. ${ }^{24}$ In addition, these enzymes often generate truncated ssDNA products (Supplementary Table S1) and yields of full-length ssDNA products, particularly for templates with stable secondary structures, have been found to be compromised. ${ }^{36}$ As an alternative to RT-based methods, we reasoned that ssDNA templates generated from asymmetrically biotinylated PCR products would produce longer ssDNA templates with higher sequence fidelity. Accordingly, we utilized an approach to generate ssDNA templates using biotin-based affinity purification of ssDNA (B-lssDNA) by exploiting the biotin-streptavidin interaction. In this method, one PCR primer used for donor amplification is biotinylated, which allows the resulting PCR product to be strand-specifically bound to streptavidin-coated beads. Subsequently, the DNA strands are separated by alkaline denaturation and the non-biotinylated strand is isolated and used as a donor for HDR (Supplementary Fig. S1C). SsDNA templates generated by all these methods were treated with $\mathrm{S} 1$ nuclease to confirm the singlestranded nature of the templates generated (Supplementary Fig. S1D). Overall, while all three approaches yielded ssDNA up to at least 3,300 bases in length, the phagemid-based approach proved to be most economical while also generating large quantities of fulllength ssDNA for use as HDR templates (Table 1).

\section{Traffic Light Reporter Multi-Cas Variant 1 (TLR-MGV1): a system to evaluate genome-editing efficiency by multiple nucleases}

Previously, Certo et al. described a traffic light reporter (TLR) system that provides positive fluorescence readouts for both error-prone DSB repair as well as precise HDR repair. ${ }^{37}$ It consists of a tandem expression cassette consisting of a "broken" GFP coding sequence followed by an out-of-frame mCherry cassette (Figure 1A). The GFP sequence is disrupted by an insertion harboring various nuclease target sites to initiate DSB formation. DSB repair by pathways such as NHEJ can result in insertions or deletions (indels) that place the downstream mCherry coding sequence in frame for productive translation (+1 frameshift). In addition, precise HDR repair of the locus can be evaluated by co-delivering a truncated GFP donor repair template with a nuclease, which will restore GFP expression while leaving the mCherry coding sequence out of frame. The fraction of GFP- and mCherry-positive cells can be rapidly measured using flow cytometry to determine editing outcomes as a function of the nuclease and donor DNA composition. We redesigned the original TLR reporter to incorporate target sites for several currently characterized nucleases (Figure 1A) by introducing protospacer adjacent motifs (PAMs) belonging to Cas9/Cas 12a orthologs from Streptococcus pyogenes (SpyCas9) ${ }^{38,}$ ${ }^{39}$, Neisseria meningiditis (Nme 1 Cas9 and Nme2Cas9) ${ }^{40-42}$, Campylobacter jejuni (CjeCas9) ${ }^{43-45}$, Staphylococcus aureus (SauCas9) ${ }^{46}$, Geobacillus stearothermophilus (GeoCas9) ${ }^{47}$, Lachnospiraceae bacterium ND2006 (LbaCas12a) ${ }^{48}$, Acidaminococcus sp. (AspCas 12a) ${ }^{48}$ and Francisella novicida (FnoCas 12). ${ }^{49}$ For several of the Cas9 orthologs (SpyCas9, Nme1Cas9, CjeCas9 
and SauCas9), DSB formation can be targeted to the exact same position. We also incorporated a second SpyCas9 target site on the opposite strand such that both SpyCas9 target sites will produce a DSB at the same position. Similarly, the Cas 12a orthologs have overlapping PAMs in the incorporated target site and therefore will generate staggered cuts within the same region. All of these target sites were combined into a sequence framework that lacks stop codons in the +1 reading frame to enable mCherry expression following the induction of a suitable indel. Hence, our updated reporter (TLR-MCV1) provides a useful platform for direct comparison of genome editing properties of the major RNA-guided genome editing tools described to date.

A single copy of TLR-MCV1 was introduced into HEK293T and K562 cells by lentiviral transduction. Using plasmid transfections of HEK293T cells to introduce the nucleases, guide RNA (listed in Supplementary Table S2) and a plasmid donor template (pCVLSFFV-d14GFP-Donor; Supplementary Table S3), we observed that all the Cas9/Cas12a sites can be targeted by the cognate nucleases to induce precise and imprecise genome editing in mammalian cells (Supplementary Fig. S2A). The two GeoCas9-expressing plasmids produced inefficient editing, which may be due to suboptimal codon usage, or to GeoCas9's preference for higher temperatures, or both. ${ }^{47}$ We also performed a dosedependence analysis to test the potency of different nucleases (Supplementary Fig. S2B). SpyCas9 was found to be the most potent nuclease for the production of frameshifts that restore mCherry expression.

\section{Circular ssDNA donors outperform linear ssDNA donors for HDR}

TLR-MGV1 provides an ideal system for direct comparisons of different DNA donor architectures since both the NHEJ and HDR efficiencies can be measured using different Cas nucleases at the same locus. To create DSBs in cells, delivery of Cas9 or Cas 12a RNPs has gained favor because these complexes can be readily electroporated into a wide variety of cell types. ${ }^{50-53}$ Furthermore, due to their rapid turnover in cells, Cas9/Cas12a RNPs display lower off-target activity than other delivery modalities without compromising on-target editing activity, thereby significantly improving the specificity of targeted genomic modifications. ${ }^{51,54}$ Delivery of SpyCas9 protein complexed with its guide RNA (SpyCas9 RNPs), or each of the three Cas12a orthologs as RNPs, proved highly effective at editing the TLR-MCV1 reporter, with indel efficiencies greater than $70 \%$ achieved as measured by $\operatorname{TIDE}^{55}$ (Supplementary Fig. S3). Next, we tested different types of ssDNA donors or a plasmid donor with SpyCas9 and AspCas 12a RNPs. As shown in Figure 1B, cssDNA elicited higher HDR efficiencies relative to equimolar quantities of linear ssDNA donors or the plasmid donor in both K562 and HEK293T cells. Using cssDNA, we achieved a statistically significant $\sim 2$-fold increase in HDR yields compared to lssDNA (Supplementary Table S4). This was true for both SpyCas9 and AspCas12a-based editing. CssDNA also achieved higher GFP integration efficiencies in comparison to plasmid donors in both K562 and HEK293T cells. Notably, we did not observe a significant difference between T-lssDNA and B-lssDNA donor efficiency in K562 cells $(p=0.0797)$, indicating that lssDNAs generated using two different approaches were largely indistinguishable once generated and purified (Supplementary Table S4). There was a statistically significant difference $(p=0.03)$ between T-lssDNA and B-lssDNA when tested in HEK293T cells with AspCas12a. However, the increase 
shown by T-lssDNA relative to B-lssDNA is modest $(<4 \%)$. Overall, among the different forms of DNA templates tested, cssDNA realized the highest HDR efficiencies.

The improved efficiency of knock-in using cssDNA may be due to increased exonuclease protection afforded by the circular nature of the ssDNA. To test this hypothesis, we circularized the lssDNA by splint-mediated ligation and tested this circularized form in TLR-MCV1 cells (Supplementary Fig. S4A). Circularization of linear ssDNA resulted in significant $(p<0.0001)$ enhancement of HDR relative to the unligated precursor in both the cell lines (Supplementary Fig. S4B, Supplementary Table S4) and comparable efficiencies to those observed with phagemid-derived cssDNA donors. This is consistent with previous studies that demonstrated improved function of end-protected nucleic acids in various cell types. $\underline{56}$

\section{Cas12a nucleases produce superior HDR yields at the TLR-MGV1 locus}

Cas 12a-based genome editing has been reported to achieve increased HDR, relative to SpyCas9, since it generates 5' overhangs and more rapidly releases the PAM-distal DNA end following cleavage. ${ }^{57}$ As shown in Figure 1B, in HEK293T cells the HDR efficiency as a fraction of total editing $([\mathrm{GFP} /(\mathrm{GFP}+\mathrm{mCherry})]$, referred to hereafter as the "HDR ratio") with all the donors tested was higher for AspCas12a compared to SpyCas9. By contrast, we did not observe increases in the HDR ratio of editing with AspCas $12 \mathrm{a}$ compared to SpyCas9 in K562 cells. To explore this observation further, we tested different orthologs of Cas12a with lssDNA and cssDNA donors. Since we had previously observed no substantial difference between B-lssDNA and T-lssDNA in HDR efficiency at the TLR-MCV1 locus, we only included T-lssDNA for the subsequent comparisons in TLR-MCV1-related experiments. Efficacy of different SpyCas9 and Cas 12a nucleases for driving HDR showed cell-line-specific differences. The LbaCas12a and FnoCas 12a variants yielded higher HDR ratios relative to SpyCas9 (Figure 1C) in both HEK293T and K562 cells. With AspCas12a, however, while HDR ratios are increased in HEK293T cells, a similar increase in HDR ratios was not observed in K562 cells. In HEK293T cells, SpyCas9 supported HDR percentages of $18 \%$ and $9.5 \%$ with cssDNA and lssDNA donors, respectively (Figure 1C, lower panel). Cas 12a orthologs increased HDR percentages to $25-31 \%$ with cssDNA template and $12-21 \%$ with linear ssDNA donor. Among the Cas12a orthologs tested, LbaCas12a and FnoCas12a showed higher HDR ratios compared to AspCas12a with cssDNA. In K562 cells, the same trends were generally observed, with the exception of editing efficiencies for AspCas12. In K562 cells, the HDR ratio increased from 0.5 with SpyCas9 to 0.7-0.8 with LbaCas 12a and FnoCas 12a when using the cssDNA donor (Supplementary Fig. S5). Thus, in these cells the HDR pathway was predominantly being harnessed for DSB repair during Cas 12amediated genome editing with the cssDNA donor. The overall HDR ratio with the linear ssDNA donor increased to approximately 0.5 with LbaCas 12 a and FnoCas 12 a (Supplementary Fig. S5). However, AspCas12a did not show similar enhancements in HDR ratio in K562 cells. Taken together these results indicate that Cas 12a orthologs may be superior for template-dependent HDR genome editing when compared to SpyCas9. 


\section{The effect of donor orientation is dependent on cell type and nuclease identity}

There are conflicting reports in the literature regarding the effect of DNA strand orientation on HDR efficiencies. A bias in HDR efficiency towards ssDNA donors that have the same sequence as the target strand (i.e. the strand base-paired to the SpyCas9 RNA guide) has been reported. ${ }^{18,58}$ However, others have not observed a significant effect of strand orientation on HDR efficiency. ${ }^{15,57,59}$ To examine strand-specific donor bias in HDR efficiencies in TLR-MGV1 cells, we generated target-strand-complementary (sense) and non-target-strand-complementary (antisense) ssDNA donors for both linear and circular DNAs and electroporated them along with SpyCas9 and AspCas12a RNPs. For both effectors, the guide RNA was complementary to the antisense strand of the TLR-MCV1 reporter. In K562-TLR-MCV1 cells, there were no significant differences between sense and antisense ssDNA donors except in the case of AspCas12a and cssDNA donors (Figure 1D). For this effector/donor combination, there was about a 2-fold increase in HDR efficiency with the sense cssDNA donor relative to antisense cssDNA donor. On the other hand, electroporated HEK293T cells exhibited higher HDR yields $(p<0.008)$ with sense cssDNA donors when used with both SpyCas9 and AspCas12a. The increase in the HDR efficiency with sense cssDNA relative to antisense cssDNA was $7 \%$ and $13 \%$ when cells were electroporated with SpyCas9 and AspCas 12a, respectively. To examine if the two different guide orientations relative to the coding region of the TLR-MCV1 sequence influence the ssDNA donor orientation preference for HDR for SpyCas9 in K562 cells, we electroporated cssDNA and lssDNA donors that were complementary to the TLR-MCV1 sense or the antisense strand, in combination with guide RNAs that were likewise complementary to either TLR-MCV1 target site strand (Supplementary Fig. S6A). We did not observe any significant differences in HDR efficiency as a function of relative guide/donor strand orientation (Supplementary Fig. S6B). Overall, while there are nuclease- and cell-type-specific differences HDR efficiencies, the relative orientation of the donor does not have a consistent impact on HDR-based editing. This is consistent with previously described ssDNA donor strand biases in HDR efficiencies, which are generally locus- and cell type-specific ${ }^{19}$.

\section{Gircular ssDNA donors are more potent than lssDNA donors for HDR}

We reasoned that the higher nuclease stability of cssDNA donors may improve the potency of cssDNA compared to lssDNA donors. To test this hypothesis, cells were electroporated with increasing amounts of ssDNA donors while keeping the amount of SpyCas9 or AspCas12a RNPs constant (Figure 1E). In K562 cells, the HDR yields peaked around 1pmol of cssDNA for both SpyCas9 and AspCas12a. We also observe severe apparent DNA toxicity at higher donor DNA concentrations $>1$ pmoles of cssDNA) resulting in reduction of HDR efficiencies. Since overall cssDNA templates are about 4-5 times longer due to the presence of the phagemid sequence elements, it's likely that DNA toxicity is associated with the total mass of DNA delivered instead of moles of DNA templates electroporated. Even so, the lssDNA donor did not perform as well as the cssDNA donor in stimulating HDR even at the highest concentration that was tested in K562 cells. The highest HDR efficiency observed for the lssDNA was about 5\% with SpyCas9 and 7\% with AspCas12a which is four and two times lower than what was 
achieved with the cssDNA donor and SpyCas9 and AspCas12a respectively. These results were also mirrored in HEK293T cells, where the cssDNA donor was more potent compared to lssDNA donor. With AspCas 12a, cssDNA reached saturation at around 2 pmols, whereas 5 pmols was needed to achieve the same effect with SpyCas9. Above these donor DNA levels, we observed a drop in HDR efficiencies, presumably due to DNA toxicity. The lssDNA donor performed poorly with SpyCas9 since the percentage of GFP-positive cells with 20 pmoles of donor was still $\sim 10 \%$ lower despite using 3-fold more moles of donor. The lssDNA performed better with AspCas12a where HDR efficiencies of $\sim 30 \%$ were achieved with 20 pmoles of lssDNA donor. However, to achieve the same HDR yields, 5-fold more moles of lssDNA was needed compared to cssDNA donor. Hence, cssDNA is more potent than lssDNA for HDR and its effect is further enhanced when employing AspCas12a as the nuclease. Collectively, the TLRMCV1-based experiments reveal that cssDNA donors are more efficient at promoting HDR repair compared to lssDNA donors.

\section{Gircular ssDNA donors provide efficient templates for fluorescent tagging of endogenous proteins}

For many functional genomic studies and gene therapy applications, targeted insertion of long DNA cassettes into endogenous loci is desirable. Most studies aimed at making targeted insertions of long DNA cassettes employ plasmid donors to provide the template for precise insertion. ${ }^{10}$ However, plasmid donors can be toxic to target cells, which makes insertion of long DNA cassettes an inefficient process in most cell types. ${ }^{16}$ To test the suitability of cssDNA for integrating larger inserts, we chose four endogenous genes in the mammalian genome based on the work of Roberts et al. ${ }^{10}$ and He et al. ${ }^{60}$ to make targeted insertions of fluorescent proteins (Figure 2A). SpyCas9 RNPs were complexed with chemically synthesized guide RNAs (listed in Supplementary Table S2) with terminal modifications to enhance intracellular stability. Electroporation of RNPs in the absence of donor DNA into HEK293T cells yielded 80-93\% indels at the four sites as measured by TIDE analysis ${ }^{55}$ (Supplementary Fig. S7), indicating efficient SpyCas9 editing of each endogenous locus. It should be noted that while guides targeting ACTB, TOMM20 and $G A P D H$ loci are complementary to the sense strand, the $S E C 61 B$ targeting guide is complementary to the antisense strand. To evaluate the relative efficiency of targeted insertion by cssDNA and lssDNA, we tagged three endogenous ORFs (SEC61B, TOMM20 and $A C T B$ ) via a direct fusion of mEGFP (Figure 2A). At the GAPDH locus, we inserted an IRES-EGFP cassette to facilitate separate expression of both gene products from the modified locus. ${ }^{60}$ To evaluate the impact of the donor cassette sequence composition on HDR efficiency, the GFP tag was replaced with a red fluorescence tag (dTomato/iTag RFP) in a corresponding donor set. Phagemid-derived cssDNA or TlssDNA donors encoding the fluorescence tag flanked by $1 \mathrm{~kb}$ homology arms were electroporated into K562 and HEK293T cells along with SpyCas9 RNPs, after which GFP- or RFP-positive cells were measured by flow cytometry to estimate the HDR-based recoding efficiency at each site of interest.

Collectively at all the loci tested, cssDNA resulted in a significantly higher frequency of functional tag integration compared to the linear T-lssDNA (Figure 2B-E; significance 370 values computed in Supplementary Table S4). Interestingly, although GFP and iTagRFP 
and dTomato fusion tags have coding sequences of similar length, we observed higher integration efficiency with GFP cssDNA donor at the ACTB and TOMM20 locus, especially in HEK293T cells, indicating that donor cassette composition may modestly influence integration efficiency in a cell type- and locus-specific manner. Similarly, at the $S E C 61 B$ locus, cssDNA mediated integration of the dTomato tag was higher than what was achieved with T-ssDNA in both K562 cells and HEK293T cells (Figure 2D). As expected, we did not observe significant differences in donor integration efficiencies between T-lssDNA and B-lssDNA donors, although variability in the efficacy was observed depending on the target site, donor composition and cell type (Supplementary Fig. S8). As with TLR-MCV1, we observed cell-type- and site-specific differences in editing efficiencies with different cssDNA donor orientations, but there was no consistent trend that defined a preferred combination of target site and donor template strand (Supplementary Fig. S9). Collectively, while we observe cell type-, locus- and donor DNA sequence- and orientation-dependent variability in DNA integration efficiencies, our results show the increased potency of cssDNA templates for tagging proteins at various endogenous genomic loci in comparison to lssDNA templates.

\section{Gircular ssDNA can effectively drive biallelic tagging of endogenous proteins}

Biallelic tagging of a target gene is often desirable for functional genomics studies, but this outcome is often hampered by low HDR efficiency. Since we observed high yields of integration with cssDNA, we tested the ability of cssDNA to support biallelic integration at various endogenous sites. To distinguish between monoallelic and biallelic integration, we electroporated equimolar amounts of cssDNA donors containing green and red fluorescent tags along with the appropriate SpyCas9 RNP into cells and measured fluorescence in these cells using flow cytometry. The majority of labeled cells expressed a single green or red fluorescent tag (Figure 3). Encouragingly, for ACTB, TOMM20 and $S E C 61 B$ loci, 17-26\% of fluorescent cells were tagged with both green fluorescent and red fluorescent proteins, indicating biallelic integration of reporter tags at these sites (Supplementary Fig. S10). Negligible levels of biallelic integration were observed at the $G A P D H$ locus, likely due to lower overall HDR efficiencies at this locus, which could reflect toxicity associated with tagging GAPDH, an essential housekeeping protein.

To further compare the efficiency of fluorescent tag integration at the genetic loci of interest using cssDNA and lssDNA, we set up a competition assay and tested different combinations of cssDNA and lssDNA donors for their abilities to insert reporter tags at the $A C T B$ locus (Figure 3B). We observed robust biallelic tagging when cssDNA donors encoding GFP and iTAGRFP tags were cotransfected in both HEK293T cells (Figure 3B) and K562 cells (Supplementary Fig. S11). Interestingly, when cssDNA was combined with an equimolar quantity of lssDNA to perform the knock-ins, we observed 30-fold higher RFP signal over GFP signal when RFP-encoding cssDNA was co-introduced with GFP-encoding lssDNA. Conversely, the combination of GFP-encoding cssDNA with RFP-encoding lssDNA yielded 10-fold more GFP-positive than RFP-positive cells. Overall these results confirm that cssDNA is more efficient than lssDNA as an HDR 417 extended coding sequence into the genome. 


\section{Discussion}

For most cellular applications, non-viral methods for the delivery of a donor DNA template are employed to achieve targeted DNA insertion at a locus of interest, owing to the ease of template production. Most previous non-viral approaches have used oligonucleotides (ODNs), plasmids or linear dsDNAs as the donor DNA template. ${ }^{10,32,61-}$ 63 More recently, long lssDNA templates have been demonstrated to provide advantages over dsDNA by both reducing toxicity to cells and increasing HDR efficiency of the DNA donor cassette. ${ }^{21,22,24}$ Enzymatic methods adopted for generating long ssDNAs have permitted the knock-in of gene segments such as fluorescent reporter tags, which are more difficult to generate as chemically synthesized donors. However, cost-effective enzymatic synthesis of long ssDNA can be challenging. In this study we performed a side-by-side comparison of cssDNA produced from phagemids with lssDNA produced either using published protocols ${ }^{22}$ or a biotin-streptavidin capture method that we utilized $^{29,} 64$ (Table 1). The biotin-affinity approach for making lssDNA permits the efficient synthesis of longer DNA templates and is not subject to the potential fidelity issues of RT-based approaches, as the lssDNA is generated entirely by high-fidelity DNA polymerases. Overall, we found that phagemid-derived cssDNA, when co-delivered with Cas9 or Cas12a RNPs, is highly effective in achieving targeted integration of DNA cassettes in mammalian cells. The production of cssDNA templates using phagemids is time- and cost-effective in comparison to methods for generating lssDNA donors, in part because it requires fewer electrophoretic or affinity purification steps.

We examined the relative efficacy of HDR potentiated by different ssDNA donor compositions in the context of different Cas nuclease effectors, relative strand orientations and donor doses. We initially assessed the effects of these parameters and the donor compositions on HDR efficiencies using a modified traffic light reporter system (TLRMCV1). This fluorescence-based system permits simultaneous evaluation of imprecise and HDR-based editing efficiencies with a range of Cas9 and Cas12a effectors. While we observed robust integration of the GFP correction cassette using SpyCas9, Cas 12a nucleases achieved higher overall yields of donor integration. The effects of ssDNA strand orientation, whether lssDNA or cssDNA, exhibited cell-line- and target-site-specific variability. Overall, the potency of cssDNA donors was significantly higher (i.e., effective at lower doses) than lssDNA donors, with the TLR-MCV1 reporter as well as at endogenous sites. When used in conjunction with SpyCas9 RNP, cssDNA-based HDR was robust even at concentrations as low as 1 pmol cssDNA donor per 100,000 cells, while lssDNA donors were 2- to 10-fold less effective at this dose. The use of large amounts of donor DNA to drive longer insertions in cell lines typically poses toxicity issues. The improved HDR potencies of cssDNA donors relative to those of the corresponding lssDNAs could arise from higher stability of these templates in cells, since the circular topology likely confers some resistance to exonucleases. Consistent with this hypothesis, post-synthetic circularization of a lss DNA template increased the HDR efficiency by about two-fold in K562 cells to levels that were comparable to phagemid-sourced cssDNA. 
465 In addition to exonuclease resistance conferred by circular topology, phagemid-derived 466 ssDNA templates offer several other advantages over lssDNA templates generated using 467 RT- or PCR-based approaches: 1) cssDNA can be generated with longer donor

468 cassettes. $^{65}$ Excluding the encoded bacterial and phage DNA sequences ( 2,200 bp), our experience indicates that DNA cassettes up to $\sim 10 \mathrm{~kb}$ (Supplementary Fig. S12) can be readily incorporated into the phagemid vector for successful ssDNA generation, without any concomitant increase in generation cost or production of truncated products. While linear ssDNA has the advantage of only containing the sequence of interest, creating donors of this length would be extremely challenging with TGIRT and potentially cumbersome even for PCR-based approaches. 2) TGIRT does not possess proofreading activity, and therefore the fidelity of ssDNA products that it produces is of concern, especially for longer donors. By contrast, the biotin-streptavidin affinity purification-based approach for generation of lssDNA and phagemid-derived cssDNA described in this paper can be used to generate accurate and full-length ssDNA. 3) The cost of generating full-length cssDNA molecules is modest compared to lssDNA generation by RT-based methods or the biotin-streptavidin affinity purification approach, which use expensive enzymes and DNA purification kits (Table 1). Moreover, the production of cssDNA can be readily scaled up to generate several micrograms of DNA at a relatively low cost, which would be cumbersome to accomplish using in vitro approaches. Overall, the efficacy of phagemid-derived cssDNAs as HDR templates, combined with their ease and economy of production, make them an attractive alternative for precise genome editing. cssDNA templates should prove advantageous for the efficient insertion of long DNA cassettes in a variety of different cell types and can be leveraged for basic science and potentially stem cell-based therapeutic applications.

\section{Methods}

\section{Plasmids}

All the plasmids generated in this study were made using standard molecular biology techniques. A list of primers used to make the donor DNA templates are listed in Supplementary Table S4. A list of plasmids created is provided in Supplementary Table S5, and plasmids have been deposited in Addgene for distribution (Deposit \#75933, 75862, 87448, and 107317).

\section{Generation of ssDNA templates using phagemids: \\ Preparation of cells}

$1 \mathrm{ml}$ of 2xYT media with $100 \mu \mathrm{g} / \mathrm{ml}$ ampicillin was inoculated with a colony of XL1-Blue cells transformed with the phagemid of interest. After culturing cells at $37^{\circ} \mathrm{C}$ for $\sim 8$ hours or until the media became slightly cloudy $\left(\mathrm{OD}_{600} \sim 0.1\right), 50 \mu \mathrm{l}$ of VCSM13 phage $\left(10^{10-11}\right.$ $\mathrm{pfu} / \mathrm{ml}$ ) was added to the bacterial culture and incubated without shaking at RT for 20 minutes. Cells were then transferred to $250 \mathrm{ml}$ 2xYT media with $100 \mu \mathrm{g} / \mathrm{ml}$ ampicillin and cultured at $37^{\circ} \mathrm{C}$ for $1-2$ hours. To select for cells that had been infected by the phage, kanamycin was added to the cells to a final concentration of $75 \mu \mathrm{g} / \mathrm{ml}$ and cultured overnight.

511 Phage pellet preparation 
512 Cells were pelleted from the media by centrifugation at 10,000g for 20 minutes. The

513 supernatant containing phage was filtered through a vacuum filter (pore size $0.22 \mu \mathrm{m}$ ) to

514 eliminate cell debris and remove any remaining bacterial cells from the supernatant.

515 DNase I (Sigma) was added to a final concentration of $10 \mu \mathrm{g} / \mathrm{ml}$ and incubated at $37^{\circ} \mathrm{C}$

516 for 3 hours to eliminate any remaining dsDNA contamination in the supernatant. $10 \mathrm{~g}$ of

517 PEG-8000 (Sigma) and $7.5 \mathrm{~g}$ of NaCl was added to $250 \mathrm{ml}$ of supernatant and incubated

518 at $4^{\circ} \mathrm{C}$ on ice for 1 to 2 hours to precipitate the phage. The supernatant was spun at

$51912,000 \mathrm{~g}$ for 30 minutes at $4^{\circ} \mathrm{C}$ and the supernatant was carefully poured out and the 
558 Generation of ssDNA templates using biotin and streptavidin-based affinity 559 purification

560 The PGR product template for producing ssDNA was generated using one unmodified and one 5'-biotinylated primer (purchased from IDT). The High-Fidelity PGR product was purified by PCR clean-up gel extraction (QIAquick Gel Extraction Kit). Streptavidin magnetic Dynabeads (NanoLink ${ }^{\text {TM }}$, catalogue number M-1002; TriLink Biotechnologies, San Diego, CA, USA) were washed and resuspended in binding solution (KilobaseBINDER ${ }^{\mathrm{TM}}$, catalogue number 60101; Invitrogen, Life Technologies) as per the manufacturer's instructions and prepared for nucleic acid binding (17 $\mu \mathrm{g}$ of biotinylated $\mathrm{dsDNA} / \mathrm{mg}$ Dynabeads, 0.8-3.3 kb). The prepared streptavidin-coated beads were incubated with biotinylated PCR product for 3 hours at room temperature or $4^{\circ} \mathrm{C}$ overnight while gently rotating the tubes to keep the beads in suspension. The supernatant was collected in an Eppendorf tube and biotinylated DNA-coated beads were separated with a magnet for 4 minutes. The beads were washed twice with buffer that consists of 50 $\mathrm{mM}$ Tris-HCl (pH 8.0), $2 \mathrm{M} \mathrm{NaCl}$ and $0.05 \%$ Tween 20 by pipetting and using a volume equivalent to the solution used for nucleic acid binding, and then the tube was placed on the magnet for 2 min to collect the beads. The beads were then washed once with buffer containing $10 \mathrm{mM}$ Tris-HCl (pH 8.0) and $50 \mathrm{mM} \mathrm{NaCl}$. The bead-containing solution was then transferred to a fresh tube and the beads were separated from the solution using a magnet for 3 minutes.

\section{Denaturation of dsDNA}

Streptavidin beads bound to the biotinylated DNA were incubated with $155 \mu \mathrm{l}$ of $0.1 \mathrm{~N}$ sodium hydroxide solution $(\mathrm{NaOH})$ for 1 minute at room temperature to achieve alkaline denaturation of the biotinylated and non-biotinylated strands of the PCR product. Biotinylated ssDNA-coated beads were then separated with a magnet for 1 minute. The supernatant was then transferred to a new $1.5 \mathrm{ml}$ tube and the tube was placed back on the magnetic stand for an additional 1 minute. The solution containing the nonbiotinylated strand was immediately neutralized by the addition of $1 \mathrm{M}$ glacial acetic acid $(15 \mu \mathrm{l}$ of $1 \mathrm{M}$ glacial acetic acid to neutralize $150 \mu \mathrm{l}$ of $0.1 \mathrm{~N} \mathrm{NaOH})$, and an equal volume of $10 \mathrm{mM}$ Tris-HCl (pH 7.5) solution was then added. The sample was applied on a Spin$\mathrm{X}$ centrifuge tube filter $(0.22 \mu \mathrm{m}$ cellulose acetate $)$ to remove any beads $(\sim 0.85 \mu \mathrm{m})$ and transferred to a fresh tube. The non-biotinylated strand was precipitated using ethanol precipitation and then re-dissolved in water.

\section{Circularization of linear ssDNA}

To circularize linear ssDNA donors generated by PCR using one 5'-phosphorylated and one 5'-biotinylated primer (IDT), the non-biotinylated and phosphorylated ssDNA was generated by the affinity purification method described above. Subsequently, phosphorylated ssDNA (e.g., $\sim 20$ pmol) was annealed with a 1.2-fold molar excess of splint oligonucleotide $(24 \mathrm{pmol})$ that spans the two ends of the ssDNA in 1x E. coli DNA ligase buffer solution (NEB) to a final volume of $200 \mu$ by heating the solution to $95^{\circ} \mathrm{C}$ for 2 minutes and then cooling the reaction on ice for 2 minutes. After annealing, 40 units of $E$. coli DNA ligase (NEB) was added to the solution and incubated at $45^{\circ} \mathrm{C}$ for 1 hour to allow ligation of the ssDNA ends to proceed to completion. The solution was then treated with 40 units of exonuclease I (NEB) and 40 units of exonuclease III (NEB) and incubated at 
$60437^{\circ} \mathrm{C}$ for $30 \mathrm{~min}$ to eliminate linear ssDNA. Exonucleases were inactivated at $70^{\circ} \mathrm{C}$ for 20 605 minutes. The cssDNA was cleaned by a NucleoSpin ${ }^{\circledR}$ (Macherey-Nagel GmbH \& Co. KG, 606 Düren, Germany) column, concentrated using ethanol precipitation, and then re-dissolved 607 in water. DNA fractions were then run on a denaturing agarose gel $(2 \%, 70 \mathrm{~V}, 2 \mathrm{hr})$ to 608 examine the integrity and purity of the cssDNA.

\section{Gell culture}

HEK293T cells were maintained in DMEM media supplemented with 10\% FBS and 1\% penicillin and streptomycin (Gibco). K562 cells were maintained in RPMI 1650 media with $1 \mathrm{mM}$ glutamine supplemented with $10 \% \mathrm{FBS}$ and penicillin and streptomycin. All the cells were maintained in a humidified incubator at $37^{\circ} \mathrm{C}$ and $5 \% \mathrm{CO}_{2}$.

\section{Electroporation of Gas9 or Gas12a RNPs}

All electroporations were done using the Neon transfection system (Invitrogen). $20 \mathrm{pmol}$ of SpyCas9-3xNLS, AspCas12a, LbaCas12a, or FnoCas12a protein, along with $25 \mathrm{pmol}$ of sgRNA (for SpyCas9) or 60 pmol of crRNA (for Cas12a), was added per reaction. Guide RNA was either generated using in vitro transcription (TLR-MCV1 locus) or was purchased from Synthego (for SpyCas9 sgRNAs targeting endogenous loci). RNP and guide RNA was precomplexed in buffer R for 10-20 minutes at room temperature and the solution was made up to a final volume of $12 \mu \mathrm{l}$. For electroporating K562 cells, 150,000-200,000 cells per reaction were used. Cells for a reaction were spun down and the media was carefully removed. Cells were resuspended in $10 \mu \mathrm{l}$ of buffer $\mathrm{R}$ containing the desired nuclease and nucleofected with 3 pulses of $1600 \mathrm{~V}$ for $10 \mathrm{~ms}$ using a $10 \mu \mathrm{l}$ Neon Tip. Cells were then plated in 24-well plates into $500 \mu$ of RPMI 1650 media supplemented with $10 \% \mathrm{FBS}$ and cultured in a humidified incubator at $37^{\circ} \mathrm{C}$ and $5 \%$ $\mathrm{CO}_{2}$ for 3-4 days for TLR experiments, and for 2 weeks for experiments with donors to knock in fluorescence tags at endogenous sites, before analysis of samples using flow cytometry. For all HDR experiments except those in Figure 1E, 1 pmol of cssDNA, linear ssDNA or plasmid donor DNA was used. Donor DNA was added to the cells resuspended in buffer R or buffer R containing Cas9/Cas12a RNP.

For experiments with HEK293T cells, roughly 100,000 cells per reaction were used and the cells were given 2 pulses of $1100 \mathrm{~V}$ for $20 \mathrm{~ms}$. For experiments shown in Figure $1 \mathrm{C}$ and 1D, 3 pmols of cssDNA, lssDNA or plasmid donor DNA were used. For the rest of the experiments except those in Figure 1E, 1 pmol of donor DNA was used for HDR experiments.

\section{FACS analysis}

Cells were first washed twice with 1x PBS before analysis using flow cytometry. All flow cytometry was performed on MACSQuant VYB by Miltenyi. For detection of mCherry signal, a yellow laser (wavelength $561 \mathrm{~nm}$ ) was used for excitation and a 615/20 nm emission filter was used. To detect GFP signal, a blue laser (excitation wavelength $488 \mathrm{~nm}$ and emission filter 525/50 nm) was used. 20,000 events were recorded for each sample and data was analyzed using Flowjo V.9.0 software. Cells were first gated on FSC-A and

649 FSC-H plot to circumscribe the single cell population. Finally, a bivariate plot between 
650 FITC-A and txRED signal was used to estimate the percentage of GFP-positive or

651 mCherry-positive population and was reported in this study as a measure of gene editing

652 or homologous recombination as applicable.

653

654

655

656

657

658

659

660

661

662

663

664

665

\section{TIDE analysis}

Genomic DNA was extracted from mammalian cells using Sigma Genelute kit or Qiagen DNeasy Blood \& Tissue Kits. PCR reactions were performed using genomic DNA as template with primers listed in Supplementary Table S4 as per the manufacturer's directions. Subsequently, PCR product was purified using the Zymo DNA purification kit and sent for analysis by Sanger sequencing along with primers listed in Supplementary Table S4. The chromatograms were analyzed with the TIDE analysis webtool ${ }^{55}$ (https://tide.nki.nl/).

\section{Cas9 and Cas12a purification}

Protein purification for 3xNLS-SpyCas9 and Cas 12a-2xNLS proteins followed a common protocol as previously described. ${ }^{66}$ The generation and characterization of the $3 \times$ NLSSpyCas9 and LbaCas12a-2xNLS constructs have been recently described. ${ }^{52,}$ 67, 68 The pET2 1a plasmid backbone (Novagen) was used to drive the expression of a hexa-Histagged version of each protein. The plasmid expressing 3xNLS-SpyCas9 (or each Cas 12a2xNLS) was transformed into E. coli Rosetta (DE3) pLysS cells (EMD Millipore) for protein production. Cells were grown at $37^{\circ} \mathrm{C}$ to an OD600 of $\sim 0.2$, then shifted to $18^{\circ} \mathrm{C}$ and induced at an OD600 of $\sim 0.4$ for 16 hours with IPTG ( $1 \mathrm{mM}$ final concentration). Following induction, cells were pelleted by centrifugation and then resuspended with $\mathrm{Ni}^{2+}$ NTA buffer [20 mM Tris-HCl (pH 7.5) + $1 \mathrm{M} \mathrm{NaCl}+20 \mathrm{mM}$ imidazole $+1 \mathrm{mM}$ TCEP] supplemented with HALT Protease Inhibitor Cocktail, EDTA-Free (100x) [ThermoFisher] and lysed with a M-110s Microfluidizer (Microfluidics) following the manufacturer's instructions. The protein was purified from the cell lysate using $\mathrm{Ni}^{2+}{ }^{2} \mathrm{NTA}$ resin, washed with five volumes of $\mathrm{Ni}^{2+}$-NTA buffer and then eluted with elution buffer [20 $\mathrm{mM}$ Tris-HCl (pH 7.5), $500 \mathrm{mM} \mathrm{NaCl}, 500 \mathrm{mM}$ imidazole, $10 \%$ glycerol]. The 3xNLSSpyCas9 (or each Cas 12a) protein was dialyzed overnight at $4^{\circ} \mathrm{C}$ in $20 \mathrm{mM}$ HEPES-NaOH (pH 7.5), $500 \mathrm{mM} \mathrm{NaCl}, 1 \mathrm{mM}$ EDTA, and $10 \%$ glycerol. Subsequently, the protein was step-dialyzed from $500 \mathrm{mM} \mathrm{NaCl}$ to $200 \mathrm{mM} \mathrm{NaCl}$ [final dialysis buffer: $20 \mathrm{mM}$ HEPES$\mathrm{NaOH}(\mathrm{pH} 7.5), 200 \mathrm{mM} \mathrm{NaCl}, 1 \mathrm{mM}$ EDTA, $10 \%$ glycerol]. Next, the protein was purified by cation exchange chromatography $[$ column $=5 \mathrm{ml}$ HiTrap-S; Buffer A $=20$ $\mathrm{mM}$ HEPES-NaOH $(\mathrm{pH}$ 7.5) $+1 \mathrm{mM}$ TCEP; Buffer $\mathrm{B}=20 \mathrm{mM}$ HEPES-NaOH $(\mathrm{pH}$ 7.5) $+1 \mathrm{M} \mathrm{NaCl}+1 \mathrm{mM}$ TCEP; flow rate $=5 \mathrm{ml} / \mathrm{min} ; \mathrm{CV}=$ column volume $=5 \mathrm{ml}$ ] followed by size-exclusion chromatography (SEC) on a Superdex-200 (16/60) column [isocratic sizeexclusion running buffer $=20 \mathrm{mM}$ HEPES-NaOH $(\mathrm{pH}$ 7.5), $150 \mathrm{mM} \mathrm{NaCl}, 1 \mathrm{mM}$ TCEP for 3xNLS-SpyCas9; or $20 \mathrm{mM}$ HEPES-NaOH (pH 7.5), $300 \mathrm{mM} \mathrm{NaCl}, 1 \mathrm{mM}$ TCEP for each Cas 12a-2xNLS]. The primary protein peak from the SEC was concentrated in an Ultra-15 Centrifugal Filters Ultracel-30K (Amicon) to a concentration around $100 \mu \mathrm{M}$ based on absorbance at $280 \mathrm{~nm}$. The purified protein quality was assessed by SDSPAGE/Coomassie staining to be $>95 \%$ pure and protein concentration was quantified with Pierce ${ }^{\mathrm{TM}}$ BCA Protein Assay Kit (ThermoFisher Scientific). Protein was stored at $80^{\circ} \mathrm{C}$ until further use.

\section{In vitro transcription}


The DNA cassette containing the U6 promoter and the sgRNA framework for SpyCas9 was cloned from pLKO1-puro vector into pBluescript SK II+ backbone. ${ }^{67}$ Plasmids expressing each guide RNA from the U6 promoter were constructed by annealing oligonucleotides encoding guide RNA and cloning it into BfuAI cleavage sites in this vector (Supplementary Table S2). Templates for in vitro transcription (IVT) of SpyCas9 guides were amplified from the cognate plasmids using NEB Q5 High-Fidelity DNA Polymerase for 30 cycles $\left(98^{\circ} \mathrm{C}, 15 \mathrm{~s} ; 65^{\circ} \mathrm{C}, 25 \mathrm{~s} ; 72^{\circ} \mathrm{C}, 20 \mathrm{~s}\right)$ using primer sets designed to include the $\mathrm{T} 7$ scaffold (Supplementary Table S4). For crRNA generation for Cas 12a orthologs, templates for in vitro transcription were generated by PCR amplification of oligonucleotides designed to include the T7 scaffold along with the guide RNA and a 15mer overlap sequence to allow annealing between the oligos (Supplementary Table S4). The oligonucleotides encoded the full-length direct repeat crRNA sequence. ${ }^{67}$ Thirty cycles of amplification were conducted using NEB Q5 High-Fidelity DNA polymerase $\left(98^{\circ} \mathrm{C}, 15 \mathrm{~s} ; 60^{\circ} \mathrm{C}, 25 \mathrm{~s} ; 72^{\circ} \mathrm{C}, 20 \mathrm{~s}\right)$. The PCR products were purified using the Zymo DNA Clean \& Concentrator Kit (Zymo Cat. \#D4005). IVT reactions were performed using the HiScribe T7 High Yield RNA Synthesis Kit using $300 \mathrm{ng}$ of PCR product as template (NEB Cat. \#E2040S). After an incubation for 16 hours at $37^{\circ} \mathrm{C}$, samples were treated with DNase I for 40 mins at $37^{\circ} \mathrm{C}$ to remove any DNA contamination. Each guide RNA was purified using the Zymo RNA Clean and Concentrator Kit. Final RNA concentration was measured using a Nanodrop instrument and stored at $-80^{\circ} \mathrm{C}$ until further use.

\section{Statistical Analysis}

R, a system for statistical computation and graphics, was used for the analysis. ${ }^{69}$

Percentage of knock-in was first arcsin-transformed to homogenize the variance. Levene's test indicates that the assumption of homogeneity of variances was met. For Figure 2B and Supplementary Fig. S8, three-way analysis of variance (ANOVA) with Completely Randomized Design was performed to test whether there were main effects of DNA topology, target gene and fluorescent tag and whether there was a gene- or/and fluorescent tag-dependent topology effect. When no significant gene- or fluorescent tagdependent topology effect was found, the main effect of DNA topology was reported. Otherwise, two levels of topology were compared within each combination of genetic loci and fluorescent tag under the ANOVA framework using the lsmeans package ${ }^{70}$ if there was a significant difference among different treatments (F-test $\mathrm{p}<0.01)$. For Figure 1D, the three primary factors considered were DNA topology, Cas type and orientation. For the other figures, two-way analysis of variance (ANOVA) with Completely Randomized Design was performed to test whether there was an overall difference among different treatment groups. When the F-test was significant $(p<0.01)$, predefined contrasts were performed within the ANOVA framework using the lsmeans package. $\mathrm{P}$ values were adjusted using the Hochberg method to correct for multiple inferences. ${ }^{71}$

\section{Author Contributions}

E.J.S., P.D.Z. and S.A.W. directed the study. S.I., A.M., E.J.S. and S.A.W. conceived the study. S.I., A.M., J.V.B., B.P.R., R.I., J.L., P.L., E.M. and J.S.B. performed experiments. L.J.Z. performed statistical analysis of data. S.I., A.M., E.J.S. and S.A.W. analyzed data. S.I., A.M., E.J.S. and S.A.W. wrote the manuscript with contributions from all authors. 


\section{Acknowledgements}

All new reagents described in this work are being deposited with the nonprofit plasmiddistribution service Addgene. This work was supported by US National Institutes of Health grants (1R01GM1 15911, Somatic Cell Genome Editing grant UG3TR002668, and 4D Nucleome grant U54DK107980) to E.J.S. and S.A.W.

\section{References}

1. Sternberg SH, Doudna JA. Expanding the Biologist's Toolkit with CRISPRAnnu Rev Biochem. 2016;85:227-264. DOI: 10.1146/annurev-biochem-060815014607 Gene-Editing Nucleases and Deaminases. Annu Rev Biochem. 2019;88:191-220. DOI: 10.1146/annurev-biochem-013118-111730

4. $\quad \mathrm{Wu} W Y$, Lebbink JHG, Kanaar R et al. Genome editing by natural and engineered CRISPR-associated nucleases. Nat Chem Biol. 2018;14:642-651. DOI: 10.1038/s41589-018-0080-x Discovered Systems Expand the CRISPR-Cas Toolkit. Mol Cell. 2017;68:15-25. DOI: 10.1016/j.molcel.2017.09.007

6. Vriend LE, Krawczyk PM. Nick-initiated homologous recombination:

768 Protecting the genome, one strand at a time. DNA Repair (Amst). 2017;50:1-13. DOI: 10.1016/j.dnarep.2016.12.005

7. Porteus MH, Baltimore D. Chimeric nucleases stimulate gene targeting in human cells. Science. 2003;300:763. DOI: 10.1126/science.1078395

8. Rothkamm K, Kruger I, Thompson LH et al. Pathways of DNA double-strand break repair during the mammalian cell cycle. Mol Cell Biol. 2003;23:5706-5715. DOI: $10.1128 / \mathrm{mcb} .23 .16 .5706-5715.2003$

7759 9. Sander JD, Joung JK. CRISPR-Cas systems for editing, regulating and targeting genomes. Nat Biotechnol. 2014;32:347-355. DOI: 10.1038/nbt.2842

10. Roberts B, Haupt A, Tucker A et al. Systematic gene tagging using CRISPR/Cas9 in human stem cells to illuminate cell organization. Mol Biol Cell. 2017;28:2854-2874. DOI: 10.1091/mbc.E17-03-0209

11. Yin $\mathrm{H}, \mathrm{Xue} W$, Chen $\mathrm{S}$ et al. Genome editing with Cas9 in adult mice corrects a disease mutation and phenotype. Nat Biotechnol. 2014;32:551-553. DOI: $10.1038 /$ nbt.2884

12. Dever DP, Bak RO, Reinisch A et al. CRISPR/Cas9 beta-globin gene targeting in human haematopoietic stem cells. Nature. 2016;539:384-389. DOI: 10.1038 /nature 20134

13. Schwank G, Koo BK, Sasselli V et al. Functional repair of CFTR by

788 Cell. 2013;13:653-658. DOI: 10.1016/j.stem.2013.11.002 
14. Bak RO, Dever DP, Reinisch A et al. Multiplexed genetic engineering of human hematopoietic stem and progenitor cells using CRISPR/Cas9 and AAV6. Elife. 2017;6. DOI: 10.7554/eLife.27873

792 15. Liang X, Potter J, Kumar S et al. Enhanced CRISPR/Cas9-mediated precise genome editing by improved design and delivery of gRNA, Cas 9 nuclease, and donor DNA. J Biotechnol. 2017;241:136-146. DOI: 10.1016/j.jbiotec.2016.11.011 16. Gaj T, Staahl BT, Rodrigues GMC et al. Targeted gene knock-in by homologydirected genome editing using Cas 9 ribonucleoprotein and AAV donor delivery. Nucleic Acids Res. 2017;45:e98. DOI: 10.1093/nar/gkx154 17. Wang $\mathrm{Y}$, Wang $\mathrm{Y}$, Chang $\mathrm{T}$ et al. Integration-defective lentiviral vector mediates efficient gene editing through homology-directed repair in human embryonic stem cells. Nucleic Acids Res. 2017;45:e29. DOI: 10.1093/nar/gkw1057 18. Richardson CD, Ray GJ, DeWitt MA et al. Enhancing homology-directed genome editing by catalytically active and inactive CRISPR-Cas9 using asymmetric donor DNA. Nat Biotechnol. 2016;34:339-344. DOI: 10.1038/nbt.3481

19. Kan Y, Ruis B, Takasugi T et al. Mechanisms of precise genome editing using oligonucleotide donors. Genome Res. 2017;27:1099-1111. DOI:

10.1101/gr.214775.116

20. Chen F, Pruett-Miller SM, Huang Y et al. High-frequency genome editing using ssDNA oligonucleotides with zinc-finger nucleases. Nat Methods. 2011;8:753-755. DOI: $10.1038 /$ nmeth.1653

21. Quadros RM, Miura H, Harms DW et al. Easi-CRISPR: a robust method for one-step generation of mice carrying conditional and insertion alleles using long ssDNA donors and CRISPR ribonucleoproteins. Genome Biol. 2017;18:92. DOI: 10.1186/s13059-017-1220-4

22. Li H, Beckman KA, Pessino V et al. Design and specificity of long ssDNA donors for CRISPR-based knock-in. bioRxiv. 2017:178905. DOI: 10.1101/178905 23. Yoshimi K, Kunihiro Y, Kaneko T et al. ssODN-mediated knock-in with CRISPR-Cas for large genomic regions in zygotes. Nat Commun. 2016;7:10431. DOI: 10.1038/ncomms10431

24. Miura H, Quadros RM, Gurumurthy CB et al. Easi-CRISPR for creating knockin and conditional knockout mouse models using long ssDNA donors. Nat Protoc. 2018;13:195-215. DOI: 10.1038/nprot.2017.153

25. Kunkel TA. Rapid and efficient site-specific mutagenesis without phenotypic selection. Proc Natl Acad Sci U S A. 1985;82:488-492.

26. Lin $\mathrm{C}$, Rinker $\mathrm{S}$, Wang X et al. In vivo cloning of artificial DNA nanostructures. Proc Natl Acad Sci U S A. 2008;105:17626-17631. DOI: 10.1073/pnas.0805416105

27. Smith GP. Filamentous fusion phage: novel expression vectors that display cloned antigens on the virion surface. Science. 1985;228:1315-1317.

28. Iyer S, Doktycz MJ. Thrombin-mediated transcriptional regulation using DNA aptamers in DNA-based cell-free protein synthesis. ACS Synth Biol. 2014;3:340-346. DOI: $10.1021 / \mathrm{sb} 4000756$

29. Mitchell LG, Merril CR. Affinity generation of single-stranded DNA for

833 1989;178:239-242. DOI: 10.1016/0003-2697(89)90631-3 
834 30. Qi H, Lu H, Qiu HJ et al. Phagemid vectors for phage display: properties, 835 characteristics and construction. J Mol Biol. 2012;417:129-143. DOI:

$836 \quad$ 10.1016/j.jmb.2012.01.038

837 31. Zhou B, Dong Q, Ma R et al. Rapid isolation of highly pure single-stranded 838 DNA from phagemids. Anal Biochem. 2009;389:177-179. DOI:

839 10.1016/j.ab.2009.03.044

$840 \quad 32$. Roth TL, Puig-Saus C, Yu R et al. Reprogramming human T cell function and 841 specificity with non-viral genome targeting. Nature. 2018;559:405-409. DOI:

842 10.1038/s41586-018-0326-5

843 33. Miura H, Gurumurthy CB, Sato T et al. CRISPR/Cas9-based generation of 844 knockdown mice by intronic insertion of artificial microRNA using longer single845 stranded DNA. Sci Rep. 2015;5:12799. DOI: 10.1038/srep12799

846 34. Leonetti MD, Sekine S, Kamiyama D et al. A scalable strategy for high847 throughput GFP tagging of endogenous human proteins. Proc Natl Acad Sci U S A. 848 2016;113:E3501-3508. DOI: 10.1073/pnas.1606731113

849 35. Xiong Y, Eickbush TH. Origin and evolution of retroelements based upon 850 their reverse transcriptase sequences. EMBO J. 1990;9:3353-3362.

851 36. Harrison GP, Mayo MS, Hunter E et al. Pausing of reverse transcriptase on 852 retroviral RNA templates is influenced by secondary structures both 5' and 3' of the 853 catalytic site. Nucleic Acids Res. 1998;26:3433-3442. DOI: 10.1093/nar/26.14.3433

854 37. Certo MT, Ryu BY, Annis JE et al. Tracking genome engineering outcome at 855 individual DNA breakpoints. Nat Methods. 2011;8:671-676. DOI:

$85610.1038 /$ nmeth.1648

857 38. Cong L, Ran FA, Cox D et al. Multiplex genome engineering using CRISPR/Cas 858 systems. Science. 2013;339:819-823. DOI: 10.1126/science.1231143

859 39. Jinek M, Chylinski K, Fonfara I et al. A programmable dual-RNA-guided DNA

860 endonuclease in adaptive bacterial immunity. Science. 2012;337:816-821. DOI:

$861 \quad 10.1126 /$ science.1225829

862 40. Hou Z, Zhang Y, Propson NE et al. Efficient genome engineering in human 863 pluripotent stem cells using Cas9 from Neisseria meningitidis. Proc Natl Acad Sci U S 864 A. 2013;110:15644-15649. DOI: $10.1073 /$ pnas. 1313587110

865 41. Esvelt KM, Mali P, Braff JL et al. Orthogonal Cas9 proteins for RNA-guided 866 gene regulation and editing. Nat Methods. 2013;10:1116-1121. DOI:

$867 \quad 10.1038 /$ nmeth.2681

868 42. Edraki A, Mir A, Ibraheim R et al. A Compact, High-Accuracy Cas9 with a 869 Dinucleotide PAM for In Vivo Genome Editing. Mol Cell. 2019;73:714-726 e714. DOI: $870 \quad 10.1016 /$ j.molcel.2018.12.003

871 43. Fonfara I, Le Rhun A, Chylinski K et al. Phylogeny of Cas 9 determines 872 functional exchangeability of dual-RNA and Cas9 among orthologous type II CRISPR873 Cas systems. Nucleic Acids Res. 2014;42:2577-2590. DOI: 10.1093/nar/gkt1074

874 44. Yamada M, Watanabe Y, Gootenberg JS et al. Crystal Structure of the Minimal 875 Cas9 from Campylobacter jejuni Reveals the Molecular Diversity in the CRISPR-Cas9 876 Systems. Mol Cell. 2017;65:1109-1121 e1103. DOI: 10.1016/j.molcel.2017.02.007

877 45. Kim E, Koo T, Park SW et al. In vivo genome editing with a small Cas9 878 orthologue derived from Campylobacter jejuni. Nat Commun. 2017;8:14500. DOI: 879 10.1038/ncomms14500 
880 46. Ran FA, Cong L, Yan WX et al. In vivo genome editing using Staphylococcus 881 aureus Cas9. Nature. 2015;520:186-191. DOI: 10.1038/nature14299

882 47. Harrington LB, Paez-Espino D, Staahl BT et al. A thermostable Cas9 with 883 increased lifetime in human plasma. Nat Commun. 2017;8:1424. DOI:

884 10.1038/s41467-017-01408-4

885 48. Zetsche B, Gootenberg JS, Abudayyeh 00 et al. Cpf1 is a single RNA-guided

886 endonuclease of a class 2 CRISPR-Cas system. Cell. 2015;163:759-771. DOI:

887 10.1016/j.cell.2015.09.038

888 49. Zetsche B, Heidenreich M, Mohanraju P et al. Multiplex gene editing by

889 CRISPR-Cpf1 using a single crRNA array. Nat Biotechnol. 2017;35:31-34. DOI:

$890 \quad 10.1038 /$ nbt.3737

891 50. DeWitt MA, Magis W, Bray NL et al. Selection-free genome editing of the

892 sickle mutation in human adult hematopoietic stem/progenitor cells. Sci Transl Med.

893 2016;8:360ra134. DOI: 10.1126/scitranslmed.aaf9336

894 51. Liang X, Potter J, Kumar S et al. Rapid and highly efficient mammalian cell

895 engineering via Cas9 protein transfection. J Biotechnol. 2015;208:44-53. DOI:

896 10.1016/j.jbiotec.2015.04.024

897 52. Xu S, Luk K, Yao Q et al. Editing aberrant splice sites efficiently restores beta-

898 globin expression in beta-thalassemia. Blood. 2019;133:2255-2262. DOI:

899 10.1182/blood-2019-01-895094

900 53. Schumann K, Lin S, Boyer E et al. Generation of knock-in primary human T

901 cells using Cas9 ribonucleoproteins. Proc Natl Acad Sci U S A. 2015;112:10437-

902 10442. DOI: $10.1073 /$ pnas.1512503112

903 54. Kim S, Kim D, Cho SW et al. Highly efficient RNA-guided genome editing in

904 human cells via delivery of purified Cas9 ribonucleoproteins. Genome Res.

905 2014;24:1012-1019. DOI: 10.1101/gr.171322.113

906 55. Brinkman EK, Chen T, Amendola M et al. Easy quantitative assessment of

907 genome editing by sequence trace decomposition. Nucleic Acids Res. 2014;42:e168.

908 DOI: $10.1093 /$ nar/gku936

909 56. Hendel A, Bak RO, Clark JT et al. Chemically modified guide RNAs enhance

910 CRISPR-Cas genome editing in human primary cells. Nat Biotechnol. 2015;33:985-

911 989. DOI: 10.1038/nbt.3290

912 57. Wang Y, Liu KI, Sutrisnoh NB et al. Systematic evaluation of CRISPR-Cas

913 systems reveals design principles for genome editing in human cells. Genome Biol.

914 2018;19:62. DOI: 10.1186/s13059-018-1445-x

915 58. Yang L, Guell M, Byrne S et al. Optimization of scarless human stem cell

916 genome editing. Nucleic Acids Res. 2013;41:9049-9061. DOI: 10.1093/nar/gkt555

917 59. Lin S, Staahl BT, Alla RK et al. Enhanced homology-directed human genome

918 engineering by controlled timing of CRISPR/Cas9 delivery. Elife. 2014;3:e04766.

919 DOI: $10.7554 /$ eLife.04766

920 60. He X, Tan C, Wang F et al. Knock-in of large reporter genes in human cells via

921 CRISPR/Cas9-induced homology-dependent and independent DNA repair. Nucleic

922 Acids Res. 2016;44:e85. DOI: 10.1093/nar/gkw064

923 61. Song F, Stieger K. Optimizing the DNA Donor Template for Homology-

924 Directed Repair of Double-Strand Breaks. Mol Ther Nucleic Acids. 2017;7:53-60. DOI:

925 10.1016/j.omtn.2017.02.006 
926 62. Zhang Y, Zhang Z, Ge W. An efficient platform for generating somatic point 927 mutations with germline transmission in the zebrafish by CRISPR/Cas9-mediated 928 gene editing. J Biol Chem. 2018;293:6611-6622. DOI: 10.1074/jbc.RA117.001080

929 63. Hayashi A, Tanaka K. Short-Homology-Mediated CRISPR/Cas9-Based Method 930 for Genome Editing in Fission Yeast. G3 (Bethesda). 2019;9:1153-1163. DOI:

931 10.1534/g3.118.200976

932 64. Uhlen M. Magnetic separation of DNA. Nature. 1989;340:733-734. DOI:

$93310.1038 / 340733 a 0$

934 65. Greenstein D, Brent R. Introduction to vectors derived from filamentous 935 phages. Curr Protoc Mol Biol. 2001;Chapter 1:Unit1 14. DOI:

936 10.1002/0471142727.mb0114s13

937 66. Iyer S, Suresh S, Guo D et al. Precise therapeutic gene correction by a simple 938 nuclease-induced double-stranded break. Nature. 2019;568:561-565. DOI:

939 10.1038/s41586-019-1076-8

940 67. Liu P, Luk K, Shin M et al. Enhanced Cas12a editing in mammalian cells and 941 zebrafish. Nucleic Acids Res. 2019. DOI: 10.1093/nar/gkz184

942 68. Wu Y, Zeng J, Roscoe BP et al. Highly efficient therapeutic gene editing of 943 human hematopoietic stem cells. Nat Med. 2019;25:776-783. DOI: 10.1038/s41591944 019-0401-y

945 69. Ihaka R, Gentleman R. R: A Language for Data Analysis and Graphics. Journal 946 of Computational and Graphical Statistics. 1996;5:299-314. DOI: 10.2307/1390807

947 70. Lenth RV. Least-Squares Means: The R Package lsmeans. 2016. 2016 (least-

948 squares means; linear models; experimental design);69:33. DOI:

$94910.18637 /$ jss.v069.i01

950 71. Huang Y, Hsu JC. Hochberg's Step-up Method: Cutting Corners off Holm's

951 Step-down Method. Biometrika. 2007;94:965-975.

952

953 


\section{Figure Legends}

955

956

957

958

959

960

961

962

963

964

965

966

967

968

969

970

971

972

973

974

975

976

977

978

979

980

981

982

983

984

985

986

987

988

989

990

991

992

993

994

995

996

997

998

999
Fig. 1. Comparisons of the activity of different DNA donors in homology-directed repair using the Traffic Light Reporter Multi-Cas Variant 1 (TLR-MCV1) cassette in human cells. (A) The schematic depicts the TLR-MCV1 system showing the SFFV promoter driving the expression of GFP and mCherry, separated by a ribosome-skipping T2A signal. The yellow arrow depicts the SFFV promoter driving the expression of the GFPT2A-mCherry cassette. The orange line indicates the insertion containing target sequences for different Cas effectors, the sequence of which is shown below the schematic of TLR-MCV1. Sequences and arrows in blue indicate overlapping PAMs and a common cut site associated with SpyCas9, Nme1Cas9, CjeCas9 and SauCas9. The bolded black sequence and black arrow depict the Nme2Cas9 PAM and cut site respectively. Magenta text shows PAMs associated with Cas12a effectors, and their approximate cut sites are shown by magenta lines. The PAMs associated with GeolCas9 and Geo2Cas9 are highlighted in green and tan text, respectively. The cut sites for these two Cas9s are shown by green and tan arrows, respectively. DSBs at any of the sites of these may be imprecisely repaired via the NHEJ pathway resulting in mCherry expression (shown on the left) if repair results in (+1 frameshift) productive translation. In the presence of donor, HDR-mediated correction of "broken" GFP region results in restoration of GFP expression (shown on the right). (B) Efficacy of distinct DNA templates in driving HDR. The graph depicts the percentage of mCherry- and GFPpositive cells obtained after co-delivery of SpyCas9 or AspCas12a RNP with cssDNA, TlssDNA, B-lssDNA or plasmid DNA repair templates into TLR-MCV1 K562 cells (upper grey box) and TLR-MCV1 HEK293T cells (lower blue box). Numbers above the bars indicate ratios of GFP-positive (shown in cyan) to total indel events [mCherry-positive (shown in red) + GFP-positive cells]. Bars represent the mean from three independent biological replicates and error bars represent the standard error of the mean (s.e.m.). (G) Comparison of cssDNA- and T-lssDNA-mediated HDR efficiency upon treatment of TLR-MCV1 cells with distinct Cas effectors. The graph depicts the percentage of mCherry- and GFP-positive cells obtained after co-delivery of SpyCas9, AspCas 12a, LbaCas12a or FnoCas12a with cssDNA and T-lssDNA DNA repair templates into TLRMCV1 K562 cells (upper grey box) and TLR-MGV1 HEK293T cells (lower blue box). Numbers above the bars indicate ratios of GFP-positive (shown in cyan) to total indel events (mCherry-positive + GFP-positive). Bars represent the mean from three independent biological replicates and error bars represent the standard error of mean (s.e.m.). (D) Effect of cssDNA and T-lssDNA donor orientation on HDR efficiency. The graph depicts the percentage of mCherry- and GFP-positive cells obtained after codelivery of SpyCas9-1 or AspCas12a (targeting the same strand) with sense (S) and antisense (AS) strand cssDNA and T-lssDNA DNA repair templates into TLR-MCV1 K562 cells (upper grey box) and TLR-MCV1 HEK293T cells (lower blue box). Numbers above the bars indicate ratios of GFP-positive (shown in cyan) to total indel events (mCherry-positive + GFP-positive). Bars represent the mean from three independent biological replicates and error bars represent s.e.m. (E) Dose dependence of cssDNA and T-lssDNA donor template-mediated HDR efficiency. The graph shows the percentage of GFP-positive cells as a function of increasing cssDNA and T-lssDNA donor DNA in the presence of SpyCas9 and AspCas12a proteins in TLR-MCV1 K562 cells (left) and 
HEK293T cells (right). Bars represent the mean from three independent biological replicates and error bars represent s.e.m.

1002

Fig. 2. Comparisons of DNA donors in homology-directed repair of endogenous genomic loci in human cells. (A) Schematic of fluorescent protein tagging. The left panel shows a schematic of a genomic region containing the SpyCas9 target site and also depicts the design of a donor template containing the fluorescent protein of interest flanked by homology arms (HA). The right panel shows a schematic of each target genomic locus and the arrangement of the fluorescent tag (EGFP, dTomato or iTagRFPT) following integration. In cases of donors delivered to fluorescently tag the GAPDH locus, the fluorescent tag is preceded by an IRES (internal ribosome entry site) and followed by a bovine growth hormone (bGH) polyadenylation sequence. (B-E) Bar graphs displaying the percentages of fluorescent cells obtained upon co-delivery of 20 pmoles of SpyCas9 complexed with 25 pmoles of guide RNA targeting the $(\mathbf{B}) A C T B$, (C) TOMM20, (D) SEC61B, or (E) GAPDH locus with or without cssDNA or T-lssDNA as a donor template. Bars represent the mean from three independent biological replicates and error bars represent s.e.m.

Fig. 3. Biallelic tagging of endogenous proteins using two different cssDNA donor templates. (A) The graph shows the percentage of fluorescent cells tagged with GFP (shown in cyan), dTomato (shown in red) or both (shown in yellow) at each locus (TOMM20, SEC61B or GAPDH) in K562 cells (top panel) and HEK293T cells (bottom panel). 20 pmol SpyCas9 RNPs were co-delivered with 0.5 pmol of each cssDNA templates. Bars represent the mean from three independent biological replicates and error bars represent s.e.m. (B) Competition between cssDNA and lssDNA templates as donors for HDR. The graph shows the percentage of cells tagged with GFP (shown in cyan), iTAG-RFP (shown in red) or both GFP and iTAG-RFP (shown in yellow) at the $A C T B$ locus. Bars represent the mean from three independent biological replicates and error bars represent s.e.m.

\section{Legends for supplementary information}

Supplementary Fig. S1. Preparation of different ssDNA templates. (A) Donor DNA is cloned into phagemid vectors containing an $\mathrm{fl}$ bacteriophage origin of replication and an antibiotic resistance marker. The plasmid is transformed into E. coli cells and superinfected with a helper phage. Depending on the orientation of the fl origin, one particular strand is packaged into phage particles and extruded into the media from which phage particles are precipitated and cssDNA is purified. (B) PCR product encoding donor DNA is generated using a 5' primer containing a T7 promoter within the tail. The product is then used as a template for in vitro transcription to generate RNA. This RNA in turn is used as a template for reverse transcription using a reverse transcriptase such as TGIRT to generate linear ssDNA (T-lssDNA). (C) A PCR primer is biotinylated at the 5' end. The resulting biotinylated PCR product is then immobilized on streptavidin magnetic beads. The immobilized PCR product is then subjected to alkaline denaturation to separate the biotinylated strand from the non-biotinylated strand. The eluted non-biotinylated DNA strand is then recovered for use as an lssDNA (B-lssDNA). 
(D) S1 nuclease digestion of DNA templates. To determine whether the templates generated are entirely single stranded, dsDNA products (Plasmid and PCR templates) and ssDNA templates (cssDNA,T-lssDNA and B-lssDNA) were digested with S1 nuclease. Undigested product ("Undig.") was loaded alongside digested products ("Dig.")

Supplementary Fig. S2. Precise and imprecise editing efficiencies for plasmid-encoded nucleases in the TLR-MCV1 reporter system. (A) Precise and imprecise editing efficacy of different Cas9 and Cas12a nucleases: The graph depicts the percentage of mCherrypositive (shown in red, representative of the indel efficiency) and GFP-positive (shown in cyan, representative of the HDR efficiency) cells obtained after co-delivery of $250 \mathrm{ng}$ plasmid-encoded nucleases, $250 \mathrm{ng}$ of gRNA plasmid and $500 \mathrm{ng}$ of plasmid donor DNA template into TLR-MGV1 HEK293T cells. Bars represent the mean from three independent biological replicates and error bars represent s.e.m. (B) Dose dependence of editing efficiency as a function of plasmid concentration: The graph depicts the percentage of mCherry-positive cells as a function of increasing concentrations of plasmids encoding various nuclease effectors while the amount of sgRNA-expressing

1064

1065

1066 plasmid was held constant. Points represent the mean from three independent biological replicates and error bars represent s.e.m.

Supplementary Fig. S3. TIDE analysis to ascertain indel efficiencies at the TLRMCV1 locus in HEK293T cells. The graph shows indel percentages observed at the TLR-MCV1 locus using SpyCas9, LbaCas12a, AspCas12a and FnoCas12a effectors based on TIDE analysis of Sanger sequencing data of the locus following nuclease treatment (in the absence of donor DNA). The green bar shows the percentage of insertions and the pink bar shows the percentage of deletions. The data show the indel percentages from three biological replicates.

1088

1089 Schematic of the approach used to generate circularized B-lssDNA. A short oligonucleotide (red) is hybridized to the B-lssDNA containing a 5'-phosphorylated end such that the oligo spans the 5' and 3' ends of the linear ssDNA. The sample is treated with $E$. coli DNA ligase to ligate the ends. The lssDNA sample is then treated with Exonucleases (I and III) to eliminate residual uncircularized lssDNA. The agarose gel shows unligated and ligated lssDNA before and after treatment with Exonucleases, which digest unprotected, linear DNA species. (B) The graph depicts the percentage of mCherry- and GFP-positive cells obtained after co-delivery of SpyCas9 with B-lssDNA and circularized B-lssDNA DNA repair templates into TLR-MGV1 K562 cells (upper grey box) and TLR-MCV1 HEK293T cells (lower blue box). Numbers above the bars indicate ratios of GFP-positive (shown in cyan) to total indels [mCherry-positive (shown in red) and GFP-positive cells]. Bars represent the mean from three independent biological replicates and error bars represent s.e.m. Numbers in the boxes below the bars show percentages of GFP-positive cells.

Supplementary Fig. S5. The ratio of GFP-positive cells to total editing in the samples shown in Figure 1C. The bar graph of the ratio of GFP-positive cells over total edited cells (mCherry-positive + GFP-positive cells) obtained upon treatment of TLR-MCV1 K562 cells (upper panel) and TLR-MCV1 HEK293T cells (lower panel) with SpyCas9, 
1094

1095

1096

1097

1098

1099

1100

1101

1102

1103

1104

1105

1106

1107

1108

1109

1110

1111

1112

1113

1114

1115

1116

1117

1118

1119

1120

1121

1122

1123

1124

1125

1126

1127

1128

1129

1130

1131

1132

1133

1134

1135

1136

1137

1138

1139

1140
AspCas 12a, LbaCas12a, or FnoCas12a in the absence of donor DNA or the presence of the indicated donor. Bars represent the mean from three independent biological replicates and error bars represent s.e.m.

Supplementary Fig. S6. Strand dependence of guide and HDR template on knock-in efficiency. (A) Schematic of guide (depicted by black and blue lines) and strand orientation relative to the TLR-MCV1 target site. The magenta carrots indicate the position of the SpyCas9 DSB. The green and red lines indicate GFP and mCherry encoding regions, respectively. The orange region depicts the small insertion containing target sites for Cas9 and Cas12a proteins. (B) The graph depicts the percentage of mCherry- and GFP-positive cells obtained after co-delivery of SpyCas9 complexed with guides (SpyCas9 RNP) targeting either strand of the TLR-MCV1 reporter along with DNA repair templates complementary to the antisense or sense strand in K562 cells (upper grey box) and HEK293T cells (lower blue box). Numbers above the bars indicate ratios of GFP-positive (shown in cyan) to total indel events [mCherry-positive (shown in red) cells and GFP-positive cells]. Bars represent the mean from three independent biological replicates and error bars represent s.e.m.

Supplementary Fig. S7. SpyCas9 gene editing efficiency at the ACTB, TOMM20, $S E C 61 B$ and GAPDH loci. Genome editing was achieved by electroporation of 20 pmoles SpyCas9 complexed with 25 pmoles of guide RNA into HEK293T cells in the absence of HDR donor. The editing percentages were calculated by TIDE analysis (indicated above the bars). Pink bars indicate the proportion of deletions and green bars indicate the proportion of insertions within the indel population.

Supplementary Fig. S8. Efficiencies of fluorescent tag integration achieved with lssDNA donors generated using the TGIRT-mediated RT-PCR (T-lssDNA) or biotinstreptavidin affinity purification (B-lssDNA) approaches. Editing efficiencies for SpyCas9 RNPs and lssDNA donor delivery targeting the $(\mathbf{A})$ ACTB, (B) TOMM20, (C) SEC61B, and (D) GAPDH loci in K562 (top panel) and HEK293T (bottom panel) cells are shown. Bars represent the mean from three independent biological replicates and error bars represent s.e.m.

Supplementary Fig. S9. Effect of orientation of cssDNA on HDR efficiencies at endogenous loci. Editing efficiencies for SpyCas9 RNPs and cssDNA donor delivery targeting the (A) ACTB, (B) TOMM20, (C) SEC61B and (D) GAPDH loci in K562 cells (top panel) and HEK293T cells (bottom panel) are shown. Green bars indicate percentages of cells expressing GFP and red bars correspond to iTAG-RFP/dTomato integration events. Solid bars correspond to donor DNA in orientation 1 (ssDNA complementary to the antisense strand of the target gene) and hashed bars correspond to orientation 2 (ssDNA complementary to the sense strand of the target gene). Bars represent the mean from three independent biological replicates and error bars represent s.e.m.

Supplementary Fig. S10. Confocal images showing tagging of GFP and iTAG-RFP at the ACTB locus (top panel) and GFP and dTomato at the TOMM20 (middle panel) or SEC61B (bottom panel) loci in HEK293T cells from experiments shown in Figure 3. 
1142 Supplementary Fig. S1 . Biallelic integration of GFP and iTagRFP in K562 cells

1143 using cssDNA template. K562 cells were electroporated with 1 pmol each of GFP- and

1144 iTagRFP-encoding cssDNA templates along with 20 pmols of SpyCas9 complexed with

114525 pmols of guide RNA targeting $A C T B$. Green bars represent the percentage of GFP-

1146 positive cells, red bars represent iTagRFP-expressing cells and yellow bars represents cells

1147 expressing both GFP and iTagRFP. Bars represent the mean from three independent

1148 biological replicates and error bars represent s.e.m.

1149

1150 Supplementary Fig. S12. 1\% agarose gel image showing $1 \mathrm{~kb}$ ladder (lane 1), as well as 1151 cssDNA generated from plasmids that are $5.4 \mathrm{~kb}$ (lane 2), 6.2kb (lane 3), 8.2kb (lane 4) and $115213.6 \mathrm{~kb}$ (lane 5) in length. 
Table 1: Features of different ssDNA preparation methods

\begin{tabular}{|c|c|c|c|c|}
\hline Preparation method & $\begin{array}{c}\text { Typical } \\
\text { Yield/Prep }\end{array}$ & Cost/Prep & Time/Prep & $\begin{array}{c}\text { Maximum Length of } \\
\text { ssDNA prepared }\end{array}$ \\
\hline cssDNA & $150-200 \mu \mathrm{g}$ & $\$ 14$ & $\begin{array}{c}24 \text { hours for } \\
\text { expression; } 6 \\
\text { hours for } \\
\text { purification }\end{array}$ & $13 \mathrm{~kb}$ \\
\hline T-IssDNA & $9 \mu \mathrm{g}$ & $\$ 65$ & $\begin{array}{c}11 \text { hours for } \\
\text { purification }\end{array}$ & $3.5 \mathrm{~kb}$ \\
\hline B-IssDNA & $12 \mu \mathrm{g}$ & $\$ 109$ & $\begin{array}{c}9 \text { hours for } \\
\text { purification }\end{array}$ & $3.3 \mathrm{~kb}$ \\
\hline
\end{tabular}

cssDNA - circular ssDNA

T-lssDNA - reverse-transcription generated linear ssDNA

B-lssDNA - biotin-based affinity purified linear ssDNA 
bioRxiv preprint doi: https://doi.org/10.1101/864199; this version posted Mecember 5, 2019. The copyright holder for this preprint (which was

$\mathbf{A}$

not certified by peer review) is the author/funder. All rights reserved. No reuse allowed without permission.

Asp | Lba | Fno

Spy | Cje | Sau

5' - TTTGGCGAGACAAATCACCTGCOTCGTGGAATACGGTAAACC - 3' 3' - AAACCGCTCTGTTTAGTGGACGGAGCA|CCTTATGCCATTTGG - 5' Geo1 $\bigwedge$ Spy $\mid \mathrm{Nme1} \wedge$ Nme2
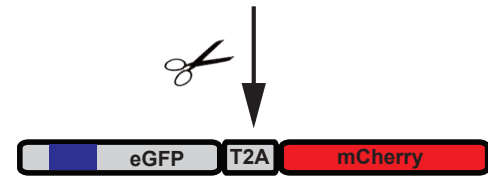

NHEJ events

\section{$\mathrm{Geo2}$}

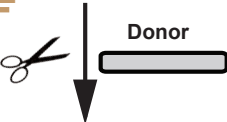

T2A mCherry

HDR events
C

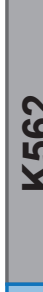

$\begin{array}{lllllllllllll}0 & 0.5 & 0.2 & 0 & 0.5 & 0.1 & 0 & 0.7 & 0.5 & 0 & 0.8 & 0.5\end{array}$

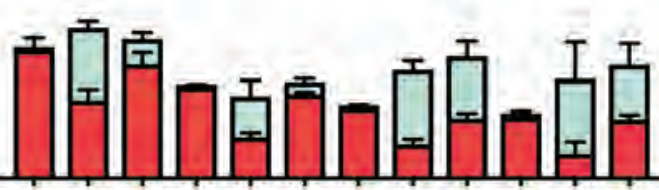

\% GFP \begin{tabular}{|l|l|l|l|l|l|l|l|l|l|l|l|l|l|l|}
\hline 0.0 & 0.3 & 0.0 & 0.1 & 16 & 5.5 & 0.2 & 8.9 & 2.6 & 0.3 & 16 & 14 & 0.4 & 16 & 12 \\
\hline
\end{tabular}

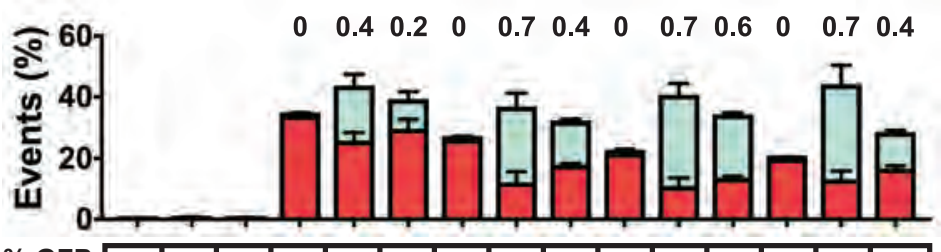

\% GFP \begin{tabular}{|l|l|l|l|l|l|l|l|l|l|l|l|l|l|l|}
\hline 0.1 & 0.4 & 0.3 & 0.3 & 18 & 9.5 & 0.3 & 25 & 14 & 0.3 & 30 & 21 & 0.3 & 31 & 12 \\
\hline
\end{tabular}

$\underset{T-1}{c}$
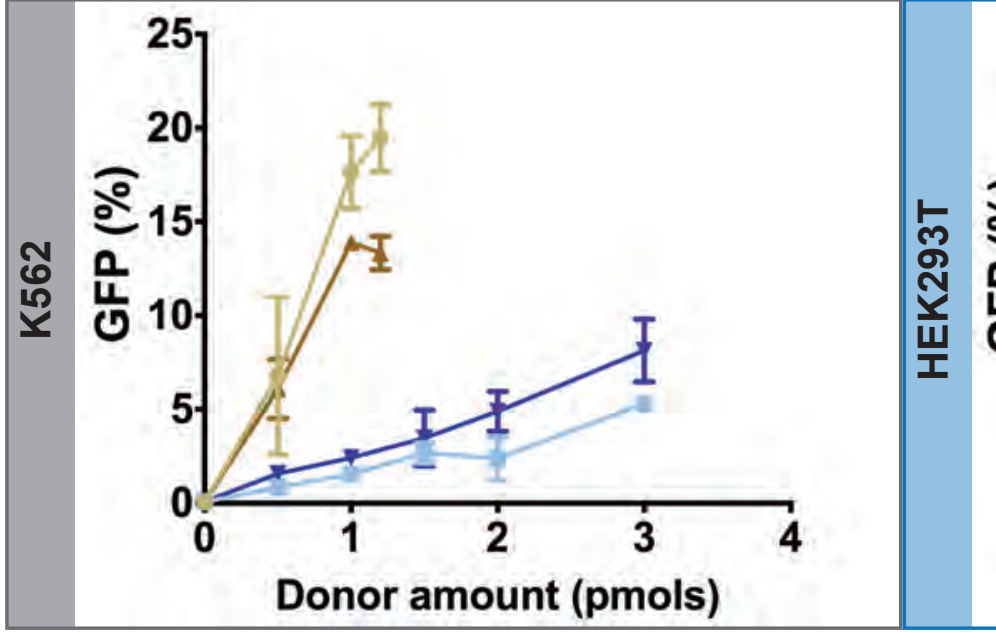

D
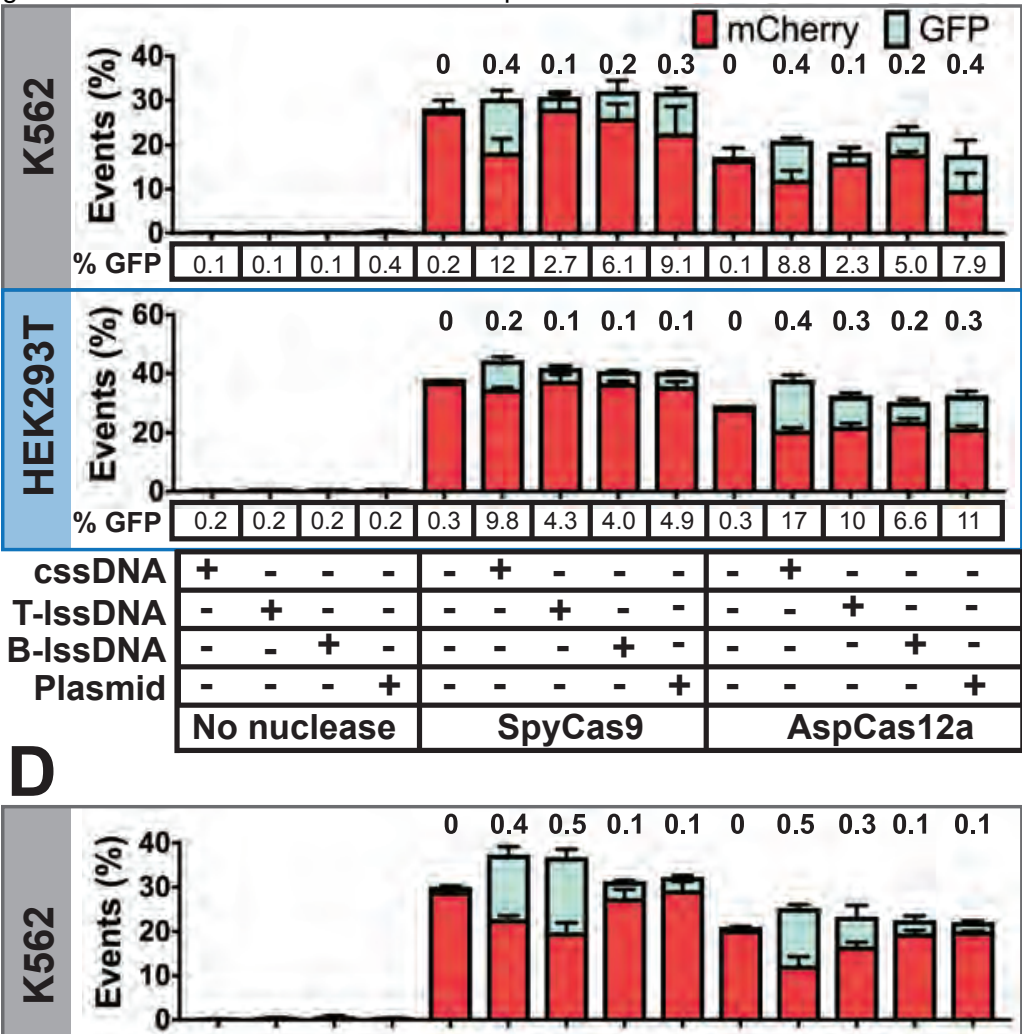

\% GFP \begin{tabular}{|l|l|l|l|l|l|l|l|l|l|l|l|l|l|}
\hline 0.1 & 0.5 & 0.3 & 0.2 & 1.0 & 15 & 17 & 3.8 & 3.0 & 0.3 & 13 & 6.7 & 3.0 & 2.1 \\
\hline
\end{tabular}

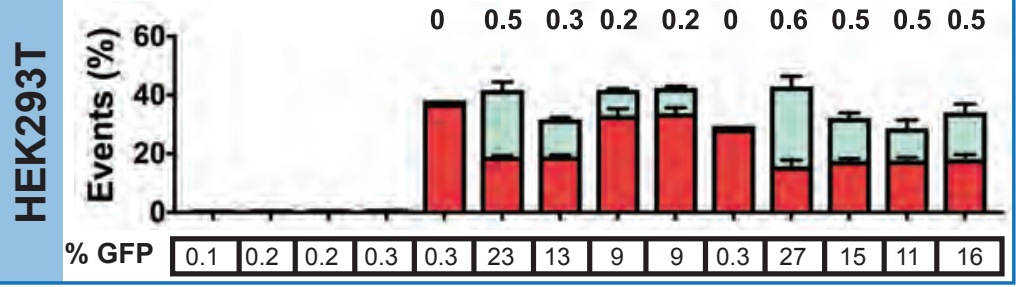

\begin{tabular}{|c|c|c|c|c|c|c|c|c|c|c|c|c|c|}
\hline \multirow{5}{*}{$\begin{array}{r}\text { cssDNAS } \\
\text { cssDNA AS } \\
\text { T-IssDNA S } \\
\text { T-IssDNA AS }\end{array}$} & + & - & - & - & + & - & $=$ & $=$ & $=$ & + & - & $=$ & - \\
\hline & $=$ & - & $=$ & $=$ & $=$ & + & $=$ & - & $=$ & - & + & - & - \\
\hline & $=$ & + & - & - & - & $=$ & + & - & $=$ & - & - & $t$ & - \\
\hline & $=$ & - & + & $=$ & - & $=$ & $=$ & + & $=$ & $=$ & - & $=$ & \pm \\
\hline & No nu & lea & & & & yc & & & & & $\mathrm{C}$ & 512 & \\
\hline
\end{tabular}

E

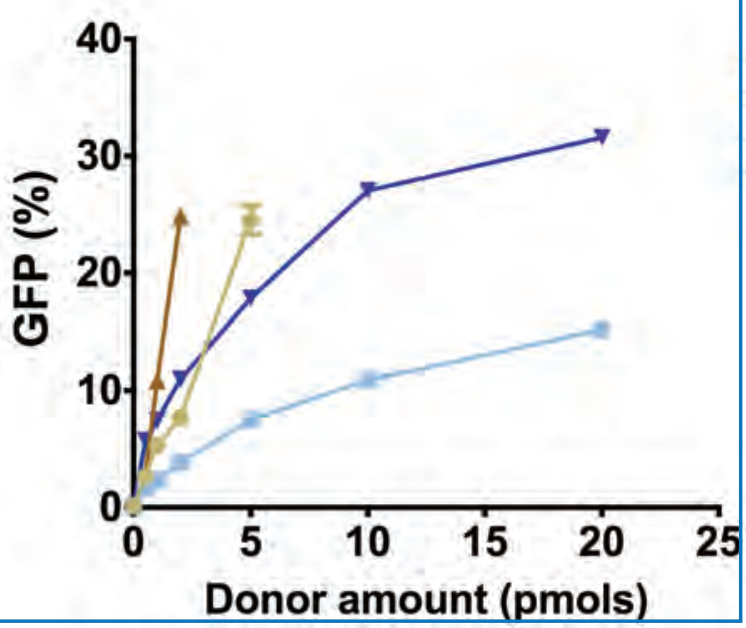

- AspCas12a cssDNA - SpyCas9 cssDNA * AspCas12a t-IssDNA SpyCas9 t-IssDNA 

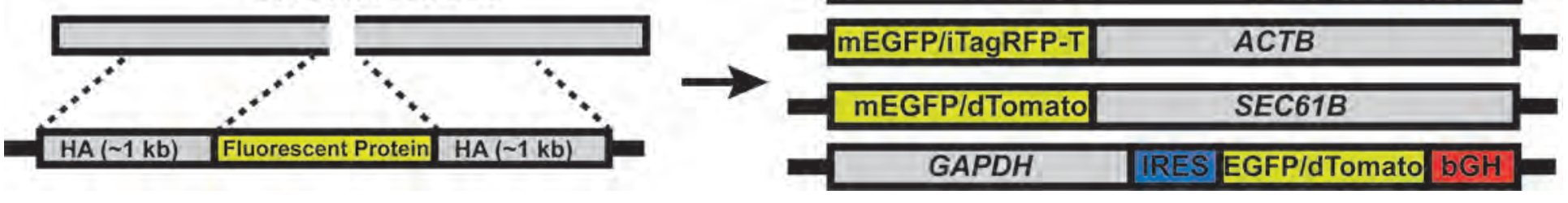

\section{ACTB}

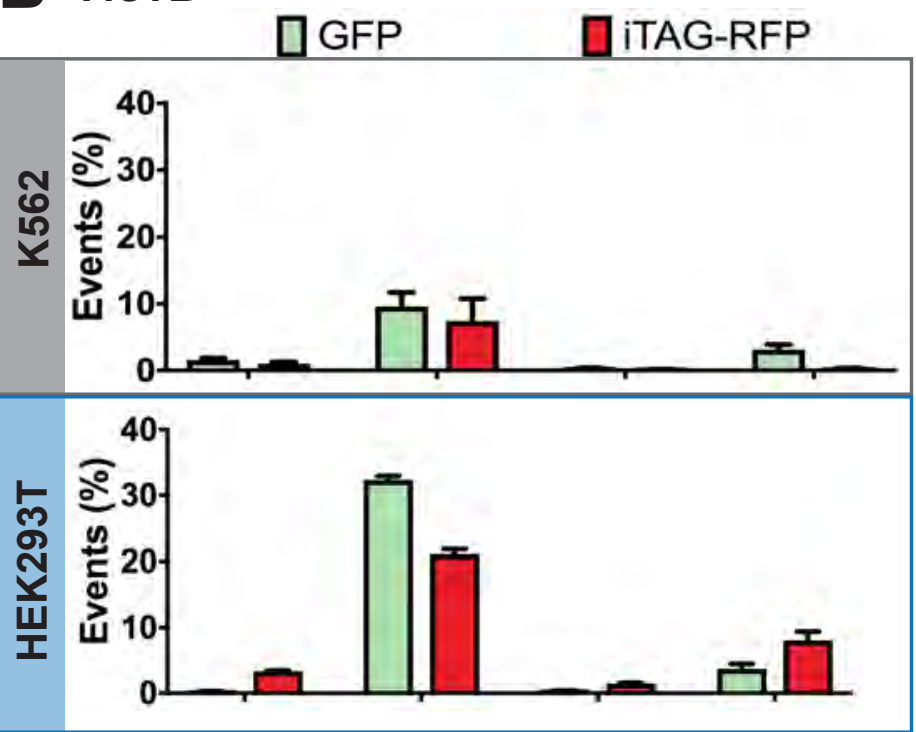

\section{SEC61B}
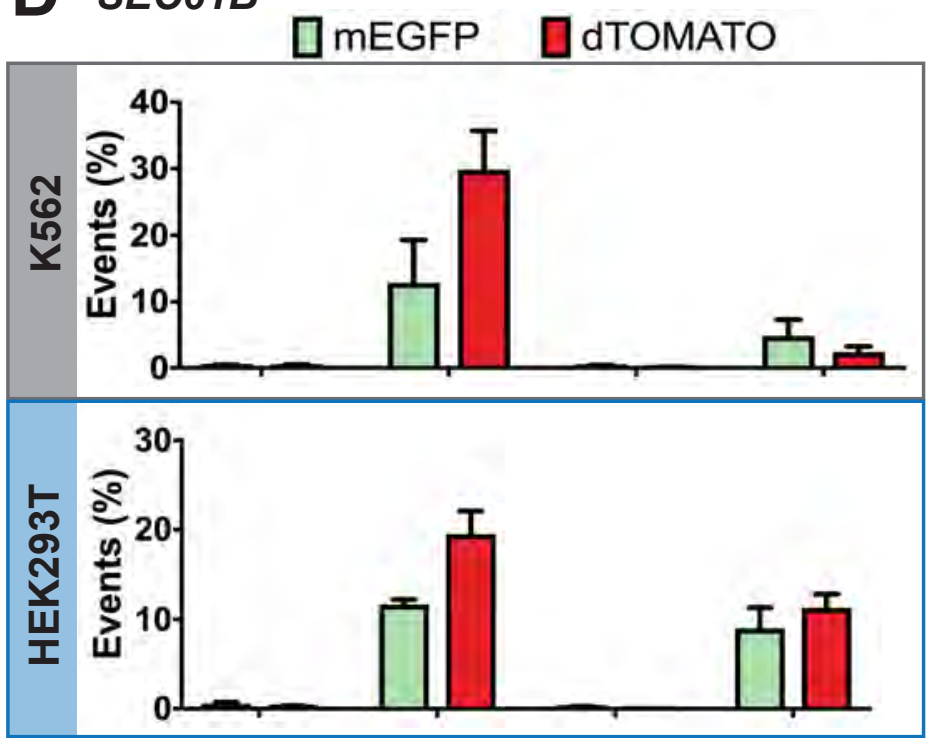

Cas9 RNP

\begin{tabular}{|c|c|}
\hline$-\quad+\quad+\quad+$ & $-\quad-\quad+\quad+$ \\
\hline cssDNA & T-IssDNA
\end{tabular}

C томм20
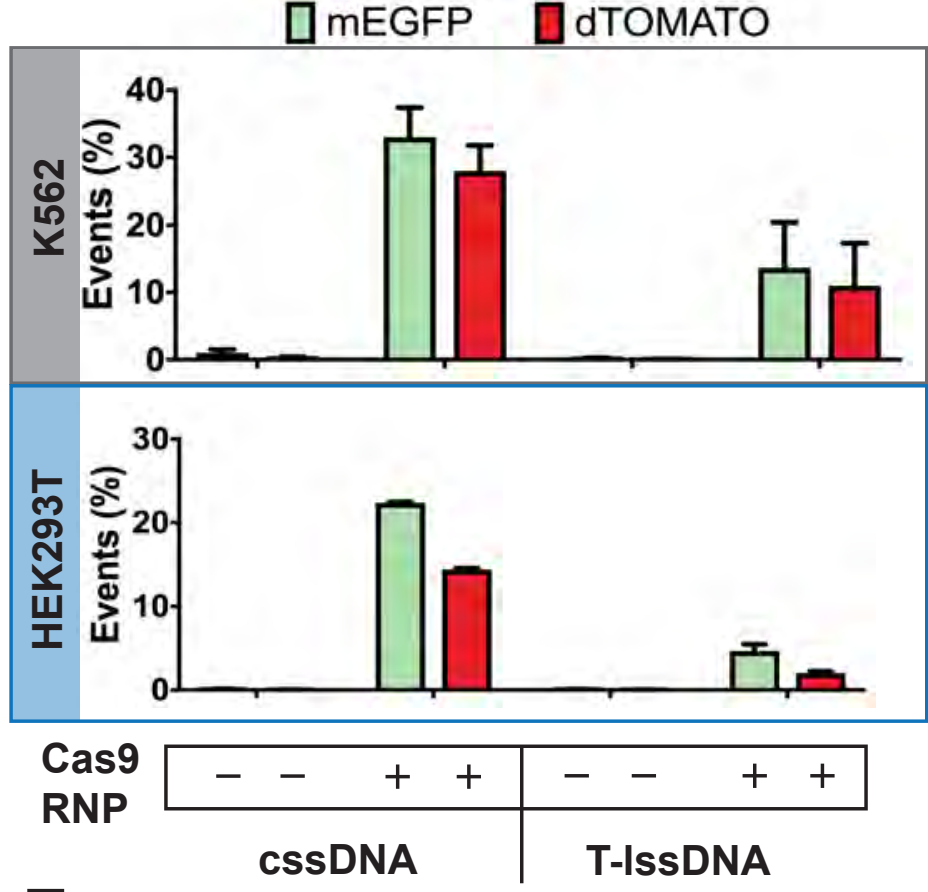

E GAPDH DMEGFP Q DTOMATO

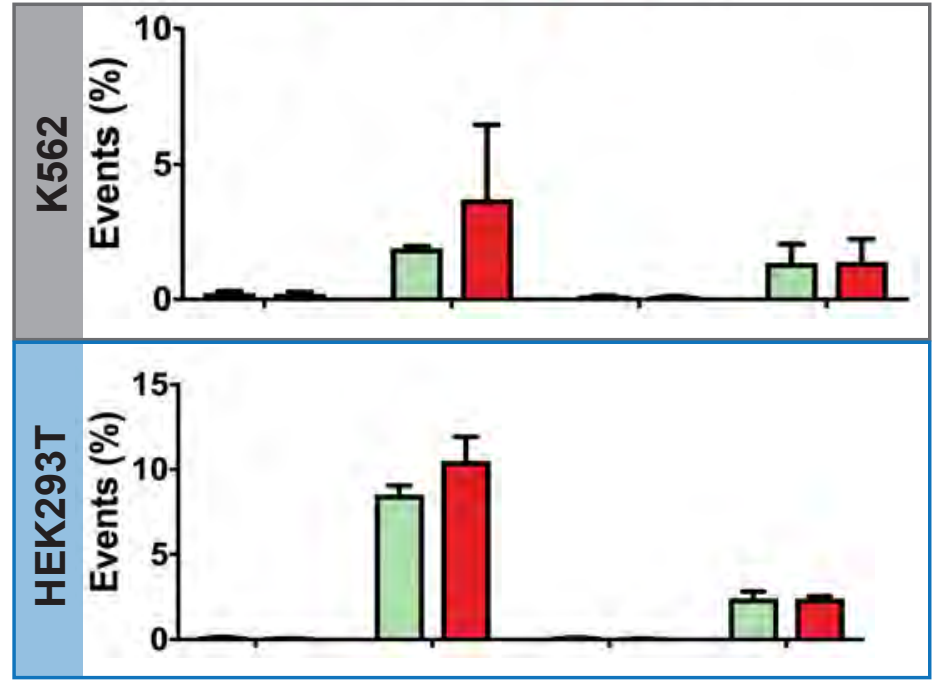

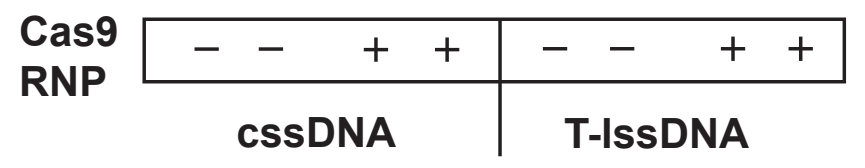




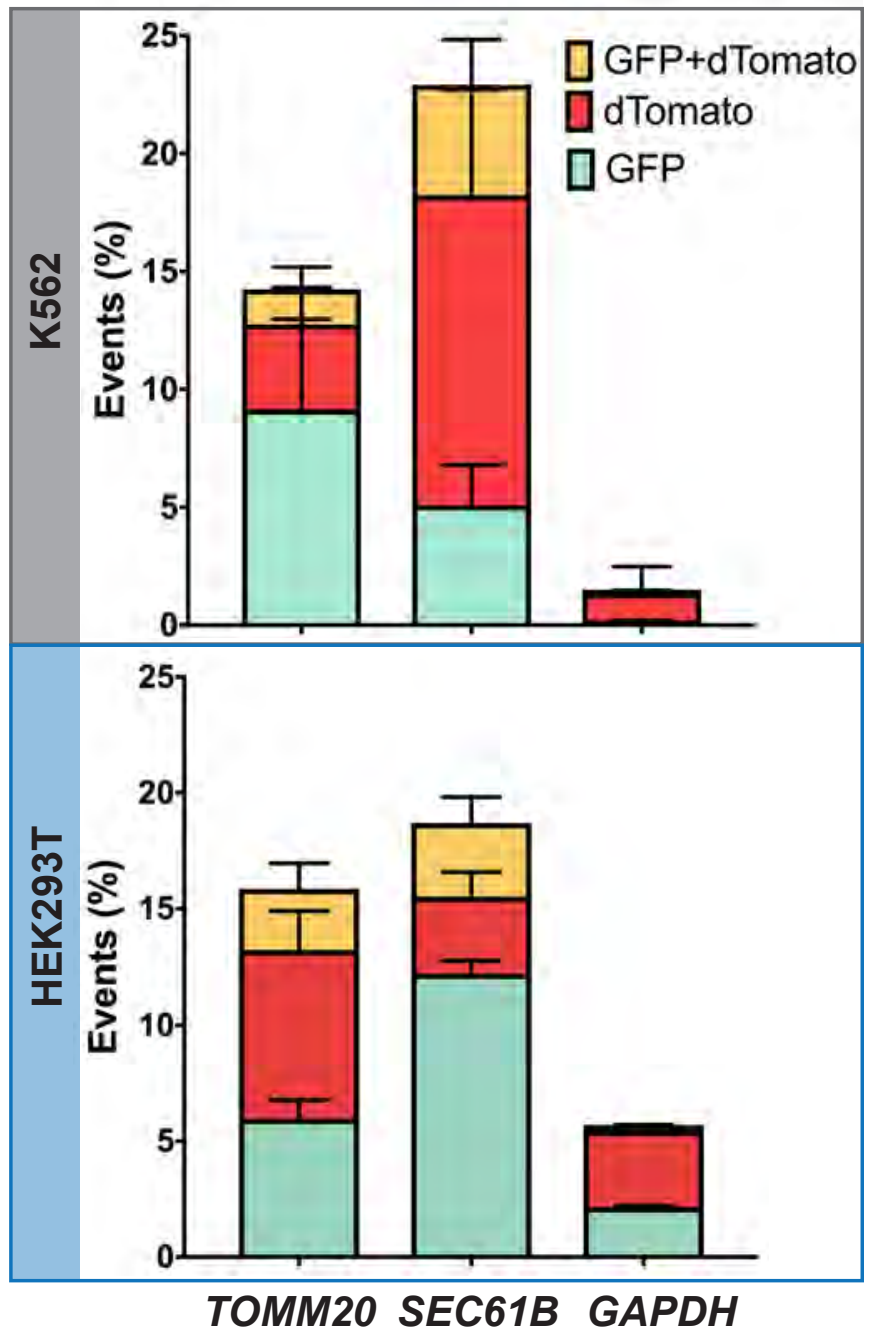

B

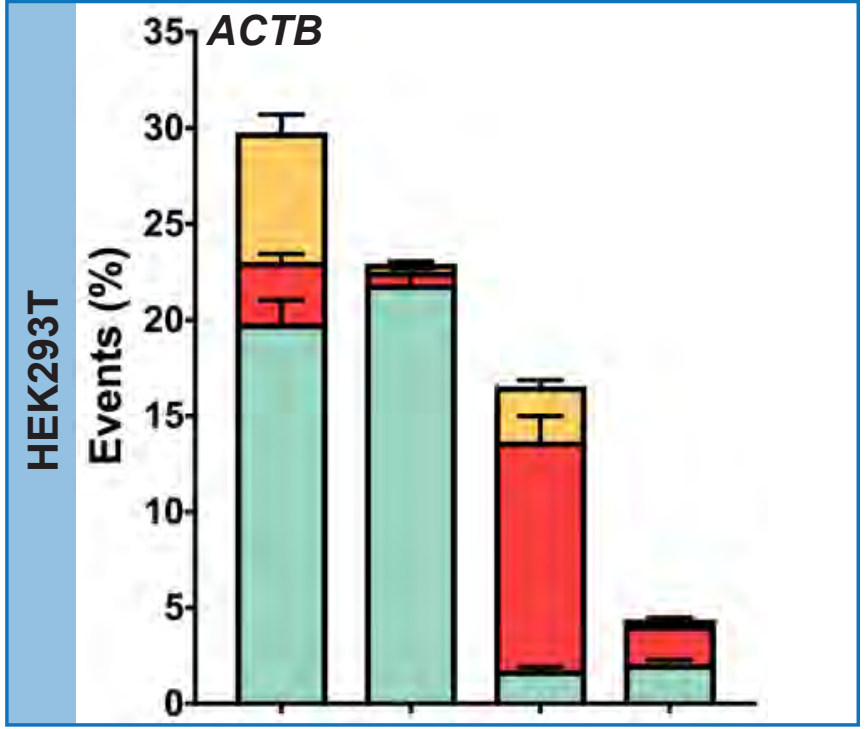

\begin{tabular}{cccc}
$+t$ & + & - & - \\
\hline+ & - & + & - \\
\hline- & - & + & + \\
\hline- & + & - & +
\end{tabular}



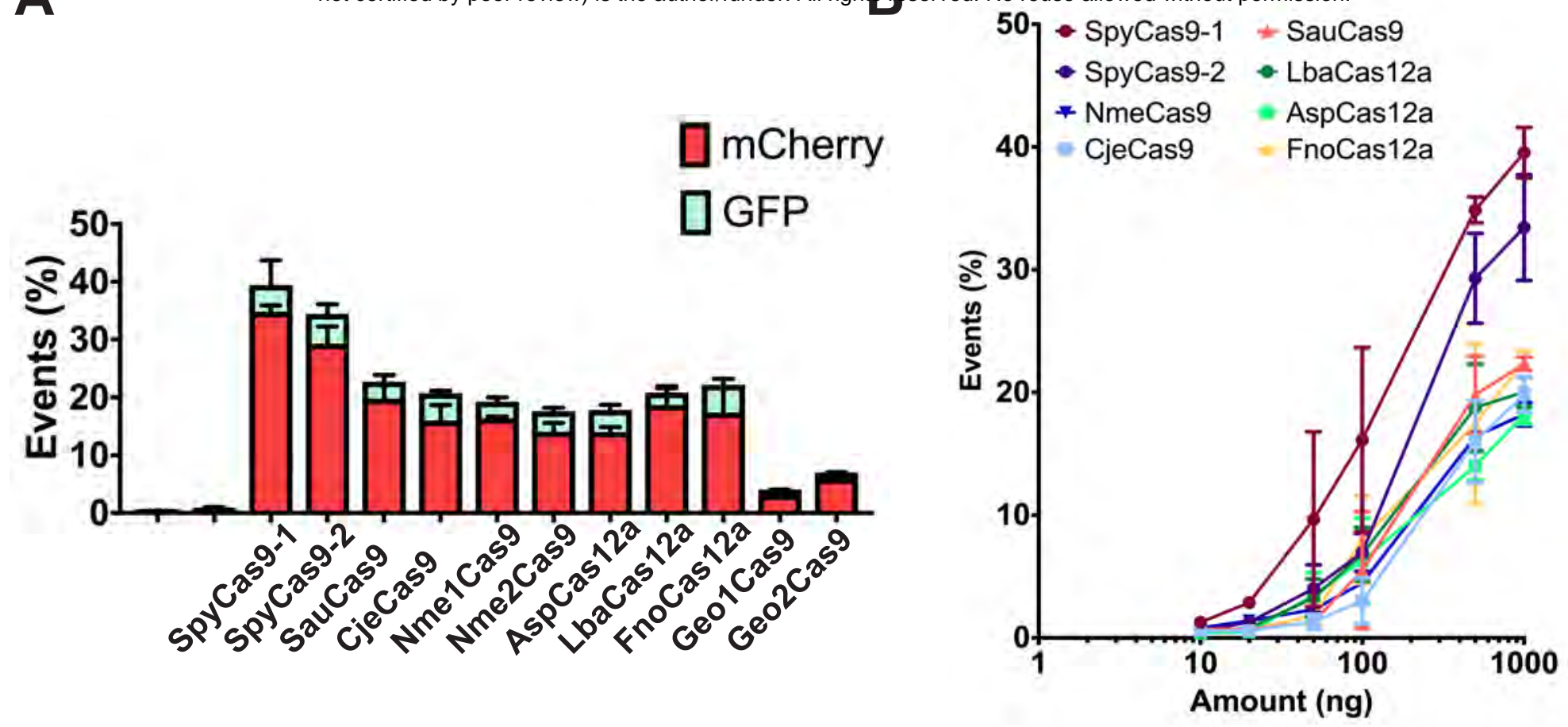


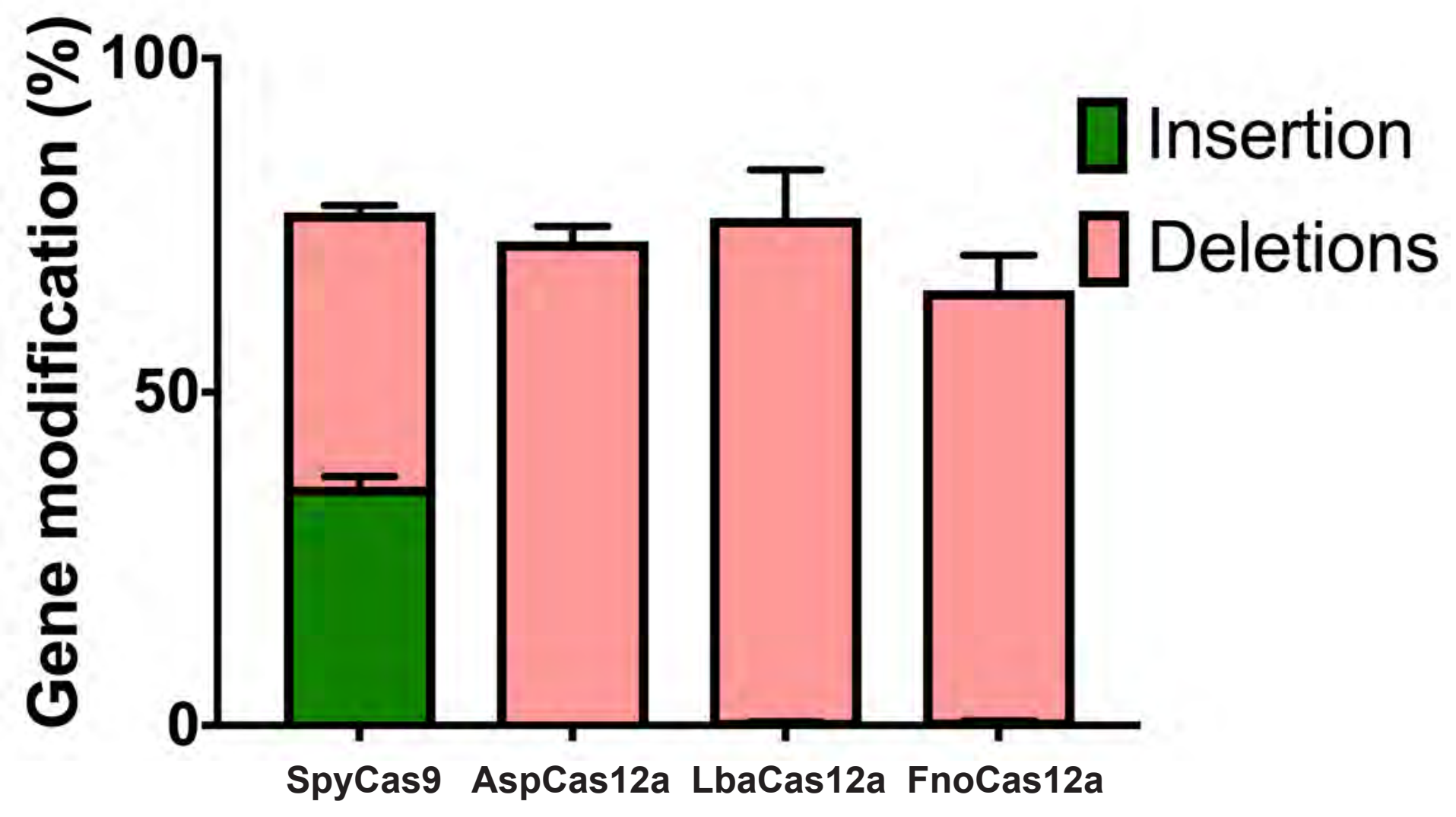

Figure S3 

not certified by peer review) is the authprifynder All rights reserved. No reuse allowed without permission.

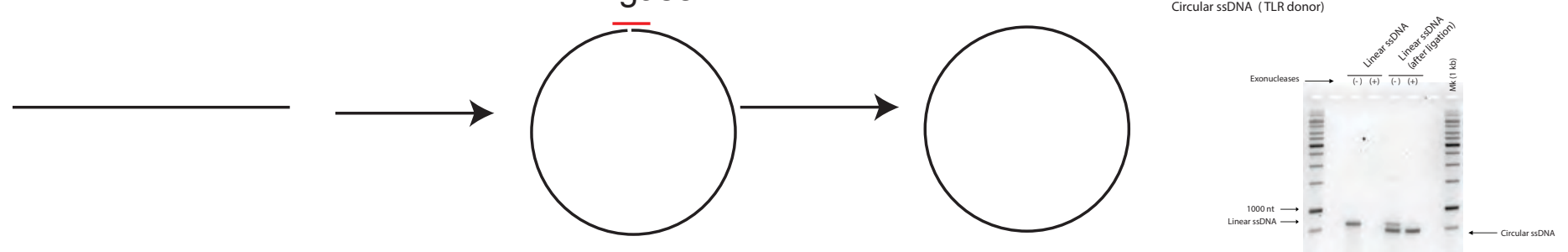

B-IssDNA

circ B-IssDNA

\section{$\square$ mCherry}

B $\quad$ QGFP
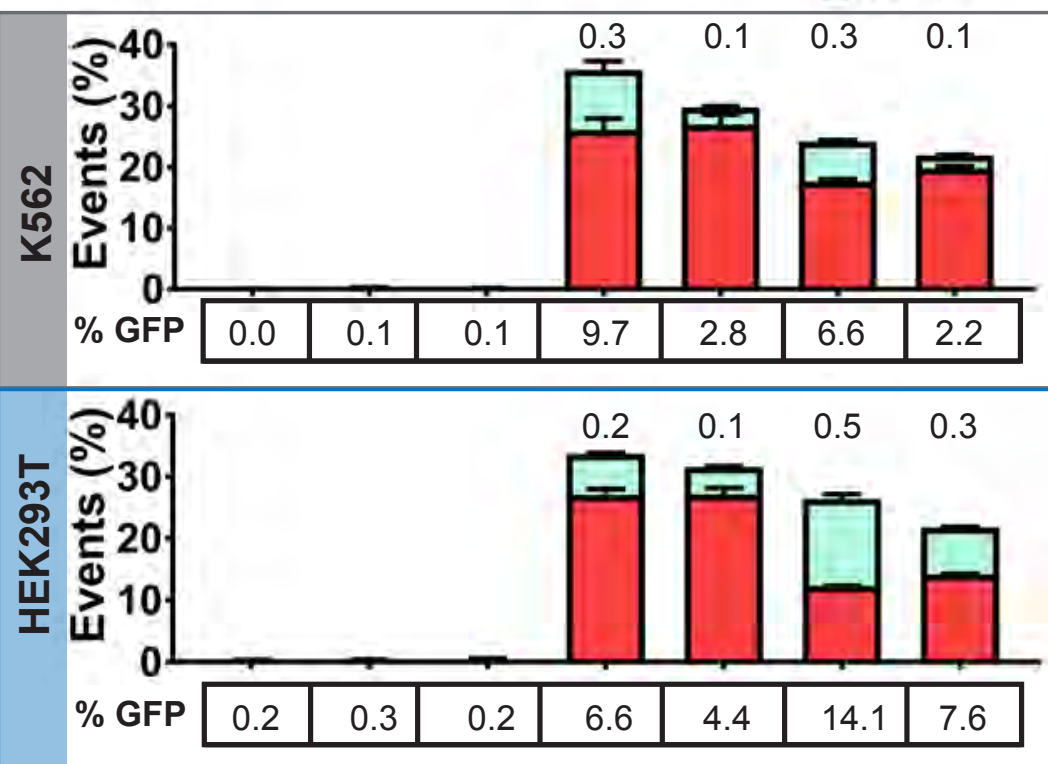

\begin{tabular}{r|c|c|c|c|c|c|c|} 
circ B-IssDNA & - & + & - & + & - & + & - \\
\cline { 2 - 8 } B-IssDNA & - & - & + & - & + & - & + \\
\cline { 2 - 7 } & \multicolumn{3}{|c|}{ No nuclease } & \multicolumn{3}{|c|}{ SpyCas9 } & \multicolumn{2}{c|}{ AspCas12a } \\
\cline { 2 - 6 }
\end{tabular}




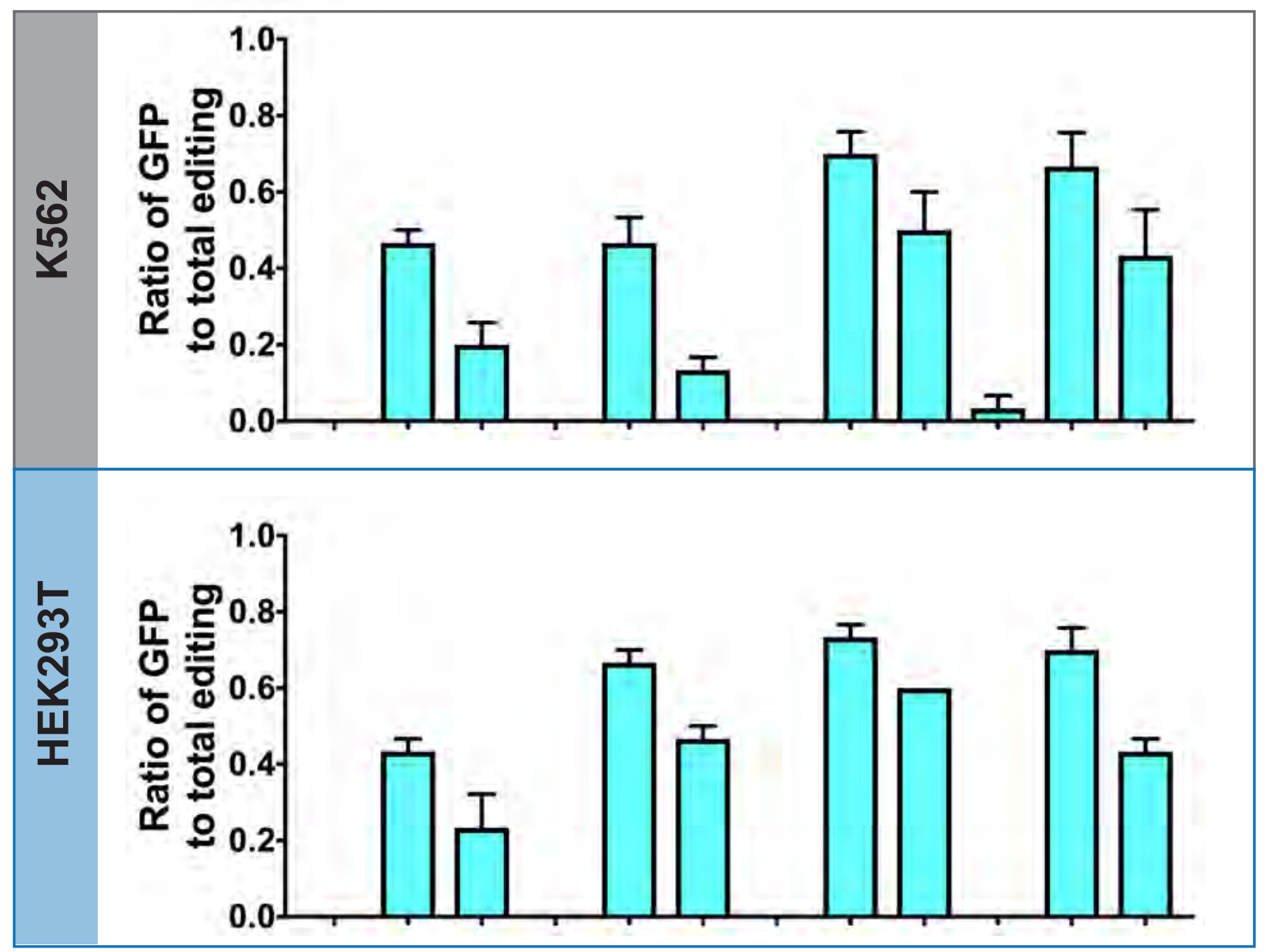

\begin{tabular}{|l|lll|lll|lll|lll|}
\hline CssDNA & - & + & - & - & + & - & - & + & - & - & + & - \\
\hline T-IssDNA & - & - & + & - & - & + & - & - & + & - & - & + \\
\hline & \multicolumn{2}{|l|}{ SpyCas9 } & AspCas12a & LbaCas12a & \multicolumn{2}{l|}{ FnoCas12a }
\end{tabular}


bioRxiv preprint doi: https://doi.org/10.1101/864199; this version posted December 5, 2019. The copyright holder for this preprint (which was not certified by peer review) is the author/funder. All rights reserved. No reuse allowed without permission.

Guide 1

Guide 2

Donor ssDNA Sense (S)

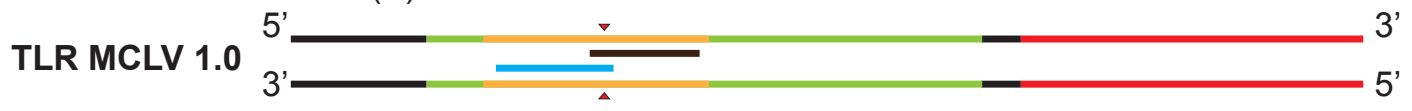

Donor ssDNA Antisense (AS)

B

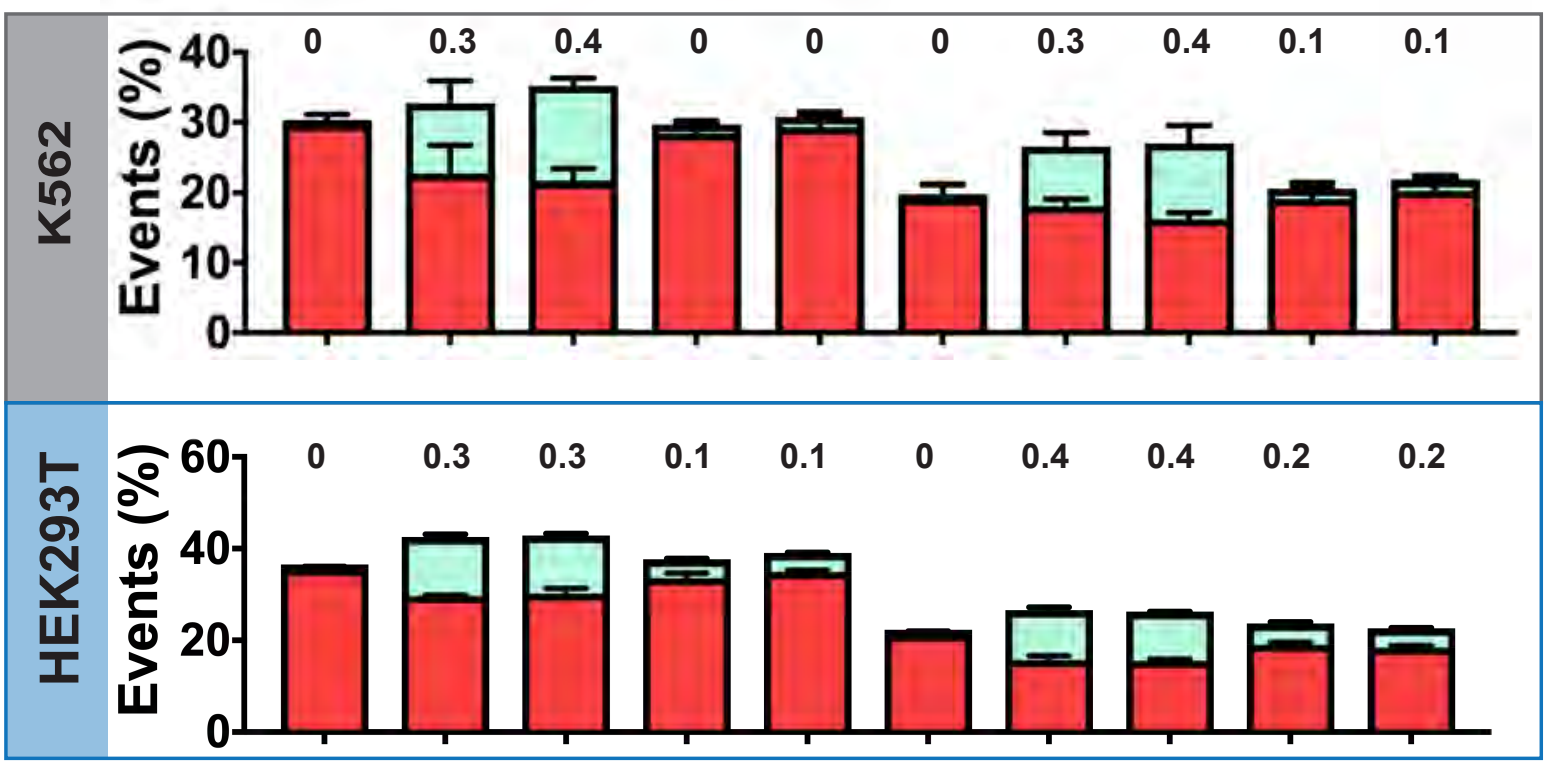

\begin{tabular}{l|l|l|l|l|l|l|l|l|l|l|}
\multirow{2}{*}{$\operatorname{css} D N A S$} \\
\cline { 2 - 12 } & - & + & - & - & - & - & + & - & - & - \\
\cline { 2 - 12 } \\
IssDNA S & - & - & + & - & - & - & - & + & - & - \\
\cline { 2 - 12 } IssDNA AS & - & - & - & + & - & - & - & - & + & - \\
\cline { 2 - 11 } & - & - & - & - & + & - & - & - & - & + \\
\hline
\end{tabular}









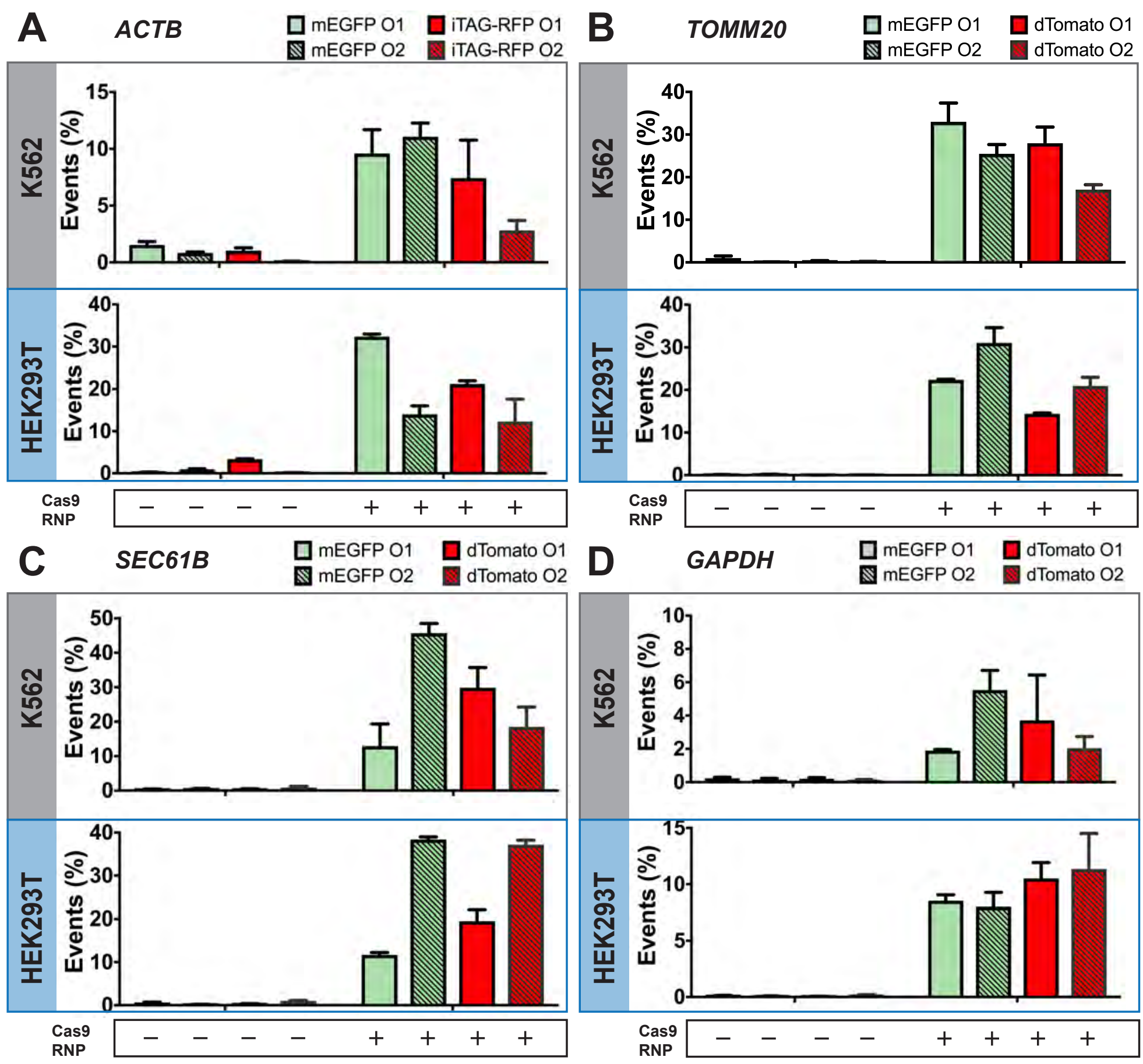

\section{Figure \$9}




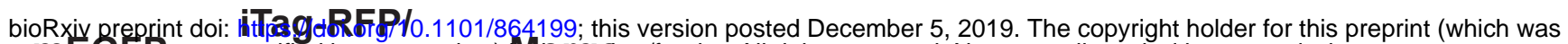
mEGFP not ceffified by peer review) Mergenor/funder. All rights reserved. No reuse allowed without permission.

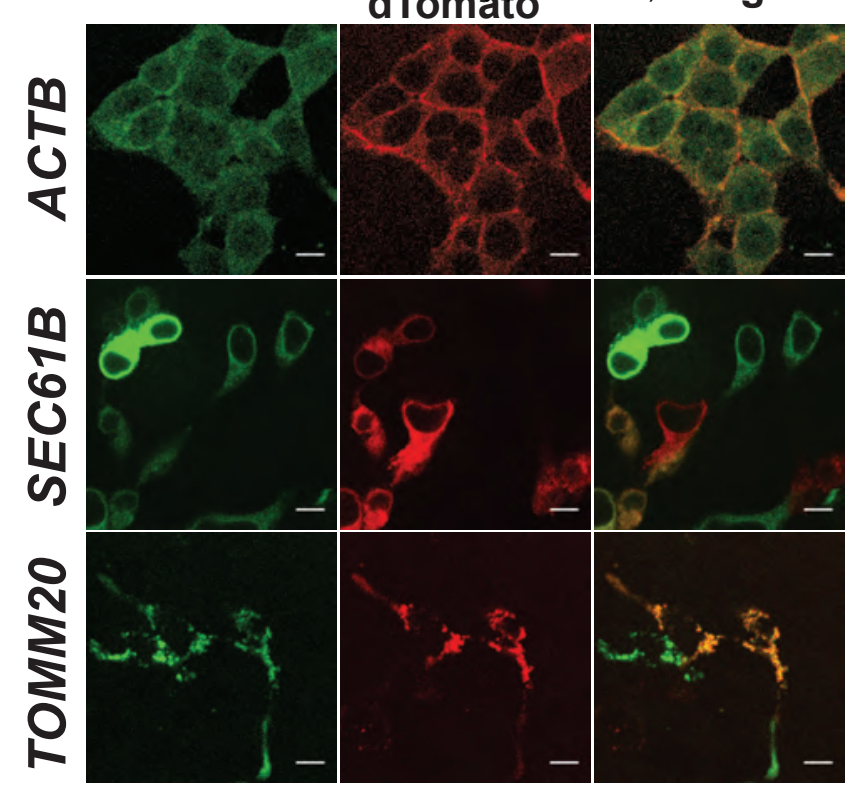

Figure S10 


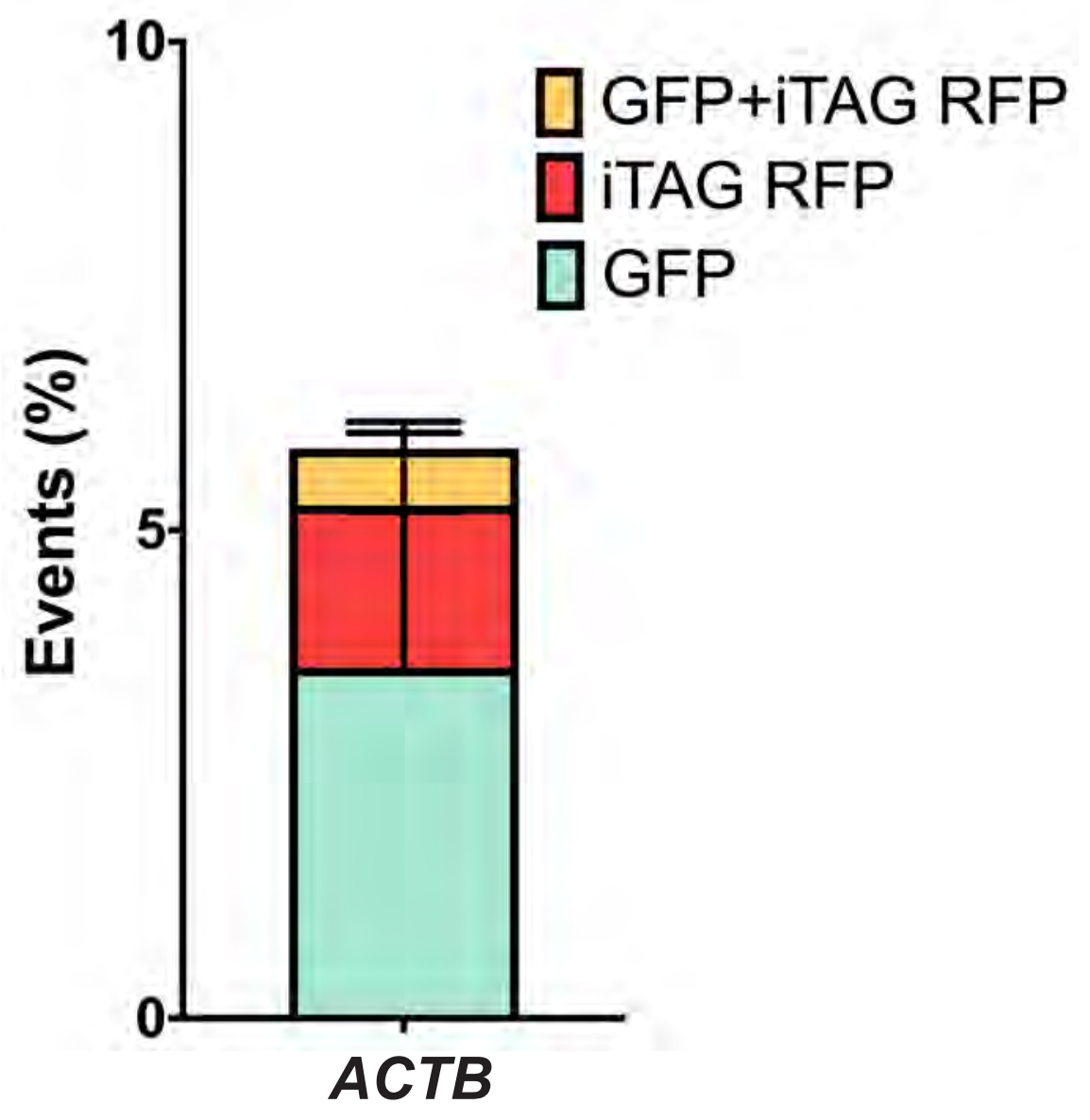

Figure S11 


\section{$1 \mathrm{~kb}$}

\section{ladder $5.4 \mathrm{~kb} 6.2 \mathrm{~kb} 8.2 \mathrm{~kb} 13.6 \mathrm{~kb}$}

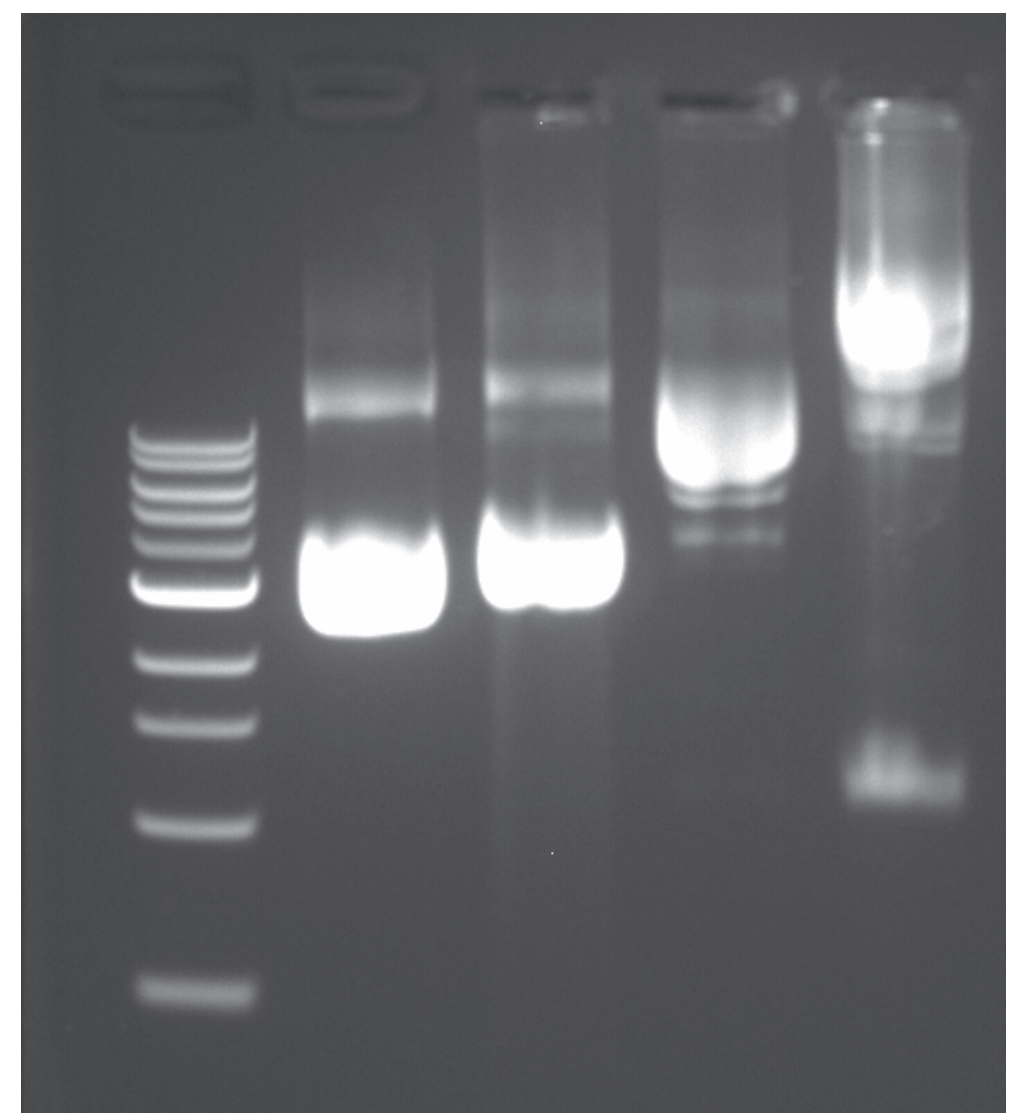

